\title{
PREPRINT
}

\section{Identification of a memory kernel in a nonlinear integrodifferential parabolic problem}

\author{
K. Van Bockstal ${ }^{\mathrm{a}, *}$, M. Slodička ${ }^{\mathrm{a}}$, F. Gistelinck ${ }^{\mathrm{b}}$ \\ ${ }^{a}$ Department of Mathematical Analysis, research group of Numerical Analysis and Mathematical Modeling $\left(\right.$ NaM $\left.{ }^{2}\right)$, \\ Ghent University, Krijgslaan 281 - S8, Ghent 9000, Belgium \\ ${ }^{b}$ Department of Data Analysis, \\ Ghent University, Henri Dunantlaan 1, Ghent 9000, Belgium
}

\begin{abstract}
In this contribution, the reconstruction of a solely time-dependent convolution kernel in an nonlinear parabolic equation is studied. The missing kernel is recovered from a global integral measurement. The existence, uniqueness and regularity of a weak solution is addressed. More specific, a numerical algorithm based on Rothe's method is designed. Numerical experiments support the obtained results.
\end{abstract}

(C) 2016 Author's personal copy

Keywords: parabolic IBVP, integral overdetermination, convolution kernel, reconstruction, convergence, time discretization

\section{Introduction}

In this contribution, the following inverse problem for a nonlinear parabolic equation with memory is considered: determine the unknown couple $\langle K, u\rangle$ obeying

$$
\left\{\begin{aligned}
\partial_{t} u(\mathbf{x}, t)-\nabla \cdot(\nabla \beta(u(\mathbf{x}, t)))+(K * u(\mathbf{x}))(t) & =\int_{0}^{t} f(u(\mathbf{x}, s)) \mathrm{d} s+F(\mathbf{x}, t), \quad \text { in } \Omega \times I, \\
-\nabla \beta(u(\mathbf{x}, t)) \cdot v & =g(\mathbf{x}, t), \quad \text { on } \Gamma \times I, \\
u(\mathbf{x}, 0) & =u_{0}(\mathbf{x}), \quad \text { in } \Omega,
\end{aligned}\right.
$$

where $\Omega$ is a bounded Lipschitz domain in $\mathbb{R}^{d}, d \geq 1$, with $\partial \Omega=\Gamma$ and $I=[0, T], T>0$, is the time frame. The usual convolution in time is denoted by $K * u$, namely $(K * u(\mathbf{x}))(t)=\int_{0}^{t} K(t-s) u(\mathbf{x}, s) \mathrm{d} s$. The goal of this paper is to develop a numerical scheme for recovering the missing time-convolution kernel $K=K(t)$. This scheme is based on

\footnotetext{
${ }^{*}$ Corresponding author

Email addresses: karel.vanbockstal@ugent. be (K. Van Bockstal), marian.slodicka@ugent.be (M. Slodička), fien.gistelinck@ugent.be (F. Gistelinck)

URL: http://cage.ugent.be/ ${ }^{\sim k v b}$ (K. Van Bockstal), http://cage.ugent.be/〜ms (M. Slodička) 
the additional integral-type measurement

$$
\int_{\Omega} u(\mathbf{x}, t) \mathrm{d} \mathbf{x}=m(t), \quad t \in[0, T] .
$$

The identification of missing memory kernels in partial integrodifferential equations of parabolic type is studied in [1, 2, 3, 4, 5]. The same subject for equations of hyperbolic type is studied in [6, 7, 8, 9, 10, 9, 11]. These papers give some local and global in time existence results for the recovery of solely time-dependent memory kernels in semilinear integrodifferential models. However, these contributions are not focussing on a numerical implementation. The development of a numerical algorithm for the kernel $K$ based on the global measurement (2) in the following semilinear parabolic problem

$$
\partial_{t} u(\mathbf{x}, t)-\Delta u+K(t) h+(K * u(\mathbf{x}))(t)=f(u, \nabla u)
$$

with Neumann-type boundary condition is done in [12]. This numerical algorithm is based on a measurement of this equation by using (2). The error analysis of the scheme is performed in [13]. The interested reader is also referred to [14], where the authors studied the same partial differential equation with a convolution of the form $K * \Delta u$ instead of $K * u$ and source term $f(u)$ instead of $f(u, \nabla u)$. More recently, in [15], using semigroup theory, the authors investigated the reconstruction of an unknown memory kernel from a more general linear integral overdetermination $\Phi(u(t))=m(t)$ in an abstract linear (of convolution type) evolution equation of parabolic type:

$$
\partial_{t} u+A u+K(t) h+(K * B u)(t)=f .
$$

In this contribution, the governing equation is nonlinear and the term $K(t) h$ is skipped out of the equation, which complicates the analysis. Crucial in the analysis is that the term depending on the solution $u$ in the right-hand side of (1) is in integral form. Note that other parameter (time-dependent) identification problems can be found in [16, 17, 18, 19, 20, 21, 22, 23, 24, 25, 26, 27, 28].

For linear $\beta$, the type of integro-differential problems under consideration arise in the theory of reactive contaminant transport [29] and in the modelling of phenomena in viscoelasticity [30]. An nonlinear application arises from the modelling of flow in variably saturated rigid soils. The water flow in porous media is usually mathematically described by the Richards equation, a nonlinear, possibly degenerate, parabolic partial differential equation. The authors in [31] derived a non-local Richards equation for the water content in the mobile domain that is characterized by a memory kernel that encodes the local mass transfer dynamics as well as the geometry of the slow zones (cf. equation [19] from [31]).

As mentioned before, the main goal of this paper is to design a numerical scheme describing a way of retrieving the couple $\langle K, u\rangle$. This is achieved not by the minimization of a cost functional (which is typical for IPs) but by the semi-discretization in time by Rothe's method. The paper is organized as follows. First, the most important notations are introduced in Section 2 and a suitable variational formulation is derived in Section 3 Section 4 deals with the numerical scheme. A priori estimates are derived in Section 5 and the convergence of the proposed numerical scheme is shown in Section 6. The uniqueness of a weak solution is addressed in Theorem 6.2. Afterwards, an error analysis is performed in Section 7 Finally, in Section 8 , the theoretically obtained results are illustrated by numerical experiments.

\section{Notations}

Denote by $(\cdot, \cdot)$ the standard inner product of $\mathrm{L}^{2}(\Omega)$ and $\|\cdot\|$ its induced norm. A similar notation is used when working at the boundary $\Gamma$, namely $(\cdot, \cdot)_{\Gamma}, \mathrm{L}^{2}(\Gamma)$ and $\|\cdot\|_{\Gamma}$. Consider an abstract Banach space $X$ with norm $\|\cdot\|_{X}$. Let $k \in \mathbb{N} \cup\{0\}$. The set of $k$-times continuously differentiable functions $w:[0, T] \rightarrow X$ equipped with the usual norm

$$
\sum_{j=0}^{k} \max _{t \in[0, T]}\left\|w^{(j)}\right\|_{X}
$$

is denoted by $\mathrm{C}^{k}([0, T], X)$. The space $\mathrm{L}^{p}((0, T), X)$ is furnished with the norm $\left(\int_{0}^{T}\|\cdot\|_{X}^{p}\right)^{\frac{1}{p}}$ with $p>1$, cf. [32]. The symbol $X^{*}$ stands for the dual space to $X$. Finally, as is usual in papers of this sort, $C, \varepsilon$ and $C_{\varepsilon}$ denote generic positive constants depending only on a priori known quantities, where $\varepsilon$ is small and $C_{\varepsilon}=C\left(\varepsilon^{-1}\right)$ is large. 


\section{Derivation of the variational problem} that

First, the PDE in (1) is multiplied with a test function $\phi \in \mathrm{H}^{1}(\Omega)$ and integrated over $\Omega$ to obtain for a.e. $t \in[0, T]$

$$
\left(\partial_{t} u(t), \phi\right)-(\nabla \cdot(\nabla \beta(u(t))), \phi)+((K * u)(t), \phi)=\left(\int_{0}^{t} f(u(s)) \mathrm{d} s, \phi\right)+(F(t), \phi) .
$$

Secondly, using Green's theorem implies that

$$
\left(\partial_{t} u(t), \phi\right)+(\nabla \beta(u(t)), \nabla \phi)+((K * u)(t), \phi)=\left(\int_{0}^{t} f(u(s)) \mathrm{d} s, \phi\right)+(F(t), \phi)-(g(t), \phi)_{\Gamma} .
$$

Now, setting $\phi=1$ in $(\mathbb{P})$ and using the measurement $\int_{\Omega} u(t)=m(t)$, it is clear that

$$
m^{\prime}(t)+(K * m)(t)=\left(\int_{0}^{t} f(u(s)) \mathrm{d} s, 1\right)+(F(t), 1)-(g(t), 1)_{\Gamma} .
$$

Finally, taking the time derivative of this equality, it holds that

$$
m^{\prime \prime}(t)+K(t) m(0)+\left(K * m^{\prime}\right)(t)=(f(u(t)), 1)+\left(F^{\prime}(t), 1\right)-\left(g^{\prime}(t), 1\right)_{\Gamma},
$$

where $F^{\prime}(t):=\partial_{t} F(t)$ and $g^{\prime}(t):=\partial_{t} g(t)$. The relations $(\mathrm{P})$ and $(\mathrm{MP})$ represent the variational formulation of the problem (1)-22). In fact, the inverse problem is reformulated into a direct one.

From now on, the real-valued everywhere differentiable function $\beta$ satisfies the properties

$$
\begin{gathered}
\quad \beta(0)=0, \\
0<\beta_{0} \leqslant \beta^{\prime}(s) \leqslant \beta_{1}, \quad \forall s \in \mathbb{R},
\end{gathered}
$$

i.e. $\beta$ is strict monotone and Lipschitz continuous. Then $\beta^{-1}$ exists and has the following properties

$$
\frac{1}{\beta_{1}} \leqslant\left(\beta^{-1}\right)^{\prime}(s) \leqslant \frac{1}{\beta_{0}}, \quad \forall s \in \mathbb{R} .
$$

The potential of $\beta$ is defined by

$$
\Phi_{\beta}(z)=\int_{0}^{z} \beta(s) \mathrm{d} s, \quad z \in \mathbb{R} .
$$

Using the properties of $\beta$, it is clear that $\Phi_{\beta}$ is convex and (see [33, Lemma 3.1])

$$
0 \leqslant \frac{\beta_{0} z^{2}}{2} \leqslant \Phi_{\beta}(z) \leqslant \frac{\beta_{1} z^{2}}{2}, \quad \forall z \in \mathbb{R} .
$$

Moreover, via the integral form of $\Phi_{\beta}$, one can easily verify by the monotone character of $\beta$ that

$$
\beta\left(z_{1}\right)\left(z_{2}-z_{1}\right) \leqslant \Phi_{\beta}\left(z_{2}\right)-\Phi_{\beta}\left(z_{1}\right) \leqslant \beta\left(z_{2}\right)\left(z_{2}-z_{1}\right), \quad \forall z_{1}, z_{2} \in \mathbb{R} .
$$

\section{Numerical scheme}

The well-posedness of $(\mathrm{P})$ and $(\mathrm{MP})$ is studied by using Rothe's method [34]. This means that a time-discrete scheme based on Backward Euler's method is designed and the convergence of the approximations towards the unique weak solution is proved under appropriate conditions on the data. Rothe's method starts with an equidistant timepartitioning of the time frame $[0, T]$ into $n \in \mathbb{N}$ intervals $\left[t_{i-1}, t_{i}\right]$. The time step is denoted by $\tau=T / n<1$ and the discrete time points by $t_{i}=i \tau, i=0, \ldots, n$. The notations

$$
z_{i} \approx z\left(t_{i}\right), \quad 0 \leqslant i \leqslant n, \quad \text { and } \quad \delta z_{i}=\frac{z_{i}-z_{i-1}}{\tau}, \quad 1 \leqslant i \leqslant n,
$$


are introduced for any function $z$. Based on $(\mathrm{P})$ and $(\overline{\mathrm{MP}})$, the following decoupled system for approximating the unknowns $(K, u)$ at time $t_{i}, 1 \leqslant i \leqslant n$, is proposed

$$
\left(\delta u_{i}, \phi\right)+\left(\nabla \beta\left(u_{i}\right), \nabla \phi\right)+\left(\sum_{k=1}^{i} K_{k} u_{i-k} \tau, \phi\right)=\left(\sum_{k=0}^{i-1} f\left(u_{k}\right) \tau, \phi\right)+\left(F_{i}, \phi\right)-\left(g_{i}, \phi\right)_{\Gamma}
$$

and

$$
m_{i}^{\prime \prime}+K_{i} m(0)+\sum_{k=1}^{i} K_{k} m_{i-k}^{\prime} \tau=\left(f\left(u_{i-1}\right), 1\right)+\left(F_{i}^{\prime}, 1\right)-\left(g_{i}^{\prime}, 1\right)_{\Gamma} .
$$

Define $v_{i}=\beta\left(u_{i}\right)$ for $i=1, \ldots, n$, then equation (DPi) can also be written as $\left\langle A\left(v_{i}\right), \phi\right\rangle=\left\langle\tilde{F}_{i}, \phi\right\rangle$, with the nonlinear operator $A: \mathrm{H}^{1}(\Omega) \rightarrow \mathrm{H}^{1}(\Omega)^{*}$ and the linear functional $\tilde{F}_{i}: \mathrm{H}^{1}(\Omega) \rightarrow \mathbb{R}$ defined by

$$
\left\langle A\left(v_{i}\right), \phi\right\rangle=\frac{1}{\tau}\left(\beta^{-1}\left(v_{i}\right), \phi\right)+\left(\nabla v_{i}, \nabla \phi\right)
$$

and

$$
\left\langle\tilde{F}_{i}, \phi\right\rangle=\frac{1}{\tau}\left(u_{i-1}, \phi\right)+\left(\sum_{k=0}^{i-1} f\left(u_{k}\right) \tau, \phi\right)+\left(F_{i}, \phi\right)-\left(g_{i}, \phi\right)_{\Gamma}-\left(\sum_{k=1}^{i} K_{k} u_{i-k} \tau, \phi\right),
$$

for any $\phi \in \mathrm{H}^{1}(\Omega)$. For given $i \in\{1, \ldots, n\}$, first (DMPi $)$ is solved and then (DPi $)$. Further, the index $i$ is increased to $i+1$. Using monotone operator theory [35], the existence and uniqueness of a solution $\left(K_{i}, u_{i}=\beta^{-1}\left(v_{i}\right)\right) \in \mathbb{R} \times \mathrm{H}^{1}(\Omega)$ for $i=1, \ldots, n$ obeying $(\overline{\mathrm{DMP} i})$ and $(\overline{\mathrm{DP} i})$ can be proved.

Theorem 4.1 (Existence of a solution on a single time step). Let $f: \mathbb{R} \rightarrow \mathbb{R}$ be bounded. The function $\beta$ satisfies 3 44. Moreover, assume that $u_{0} \in \mathrm{L}^{2}(\Omega), g \in \mathrm{C}^{1}\left([0, T], \mathrm{L}^{2}(\Gamma)\right), F \in \mathrm{C}^{1}\left([0, T], \mathrm{L}^{2}(\Omega)\right)$, and $m \in \mathrm{C}^{2}([0, T])$ with $m(0) \neq 0$. Then there exist $C>0$ and $\tau_{0}>0$ such that for any $\tau<\tau_{0}$ and each $i \in\{1, \ldots, n\}$ there exists an unique couple $\left(K_{i}, u_{i}\right) \in \mathbb{R} \times \mathrm{H}^{1}(\Omega)$ solving $(\overline{\mathrm{DMP} i})$ and $(\overline{\mathrm{DPi}})$. Moreover, there exists a positive constant $C$ such that

$$
\max _{i=1, \ldots, n}\left|K_{i}\right| \leqslant C
$$

Proof. If $m^{\prime}(0)=0$, then $\left|m(0)+m^{\prime}(0) \tau\right|>0$. If $m^{\prime}(0) \neq 0$, then set $\tau_{0}=\min \left\{1, \frac{|m(0)|}{2\left|m^{\prime}(0)\right|}\right\}$. Then for any $\tau<\tau_{0}$, we get by the triangle inequality that

$$
\left|m(0)+m^{\prime}(0) \tau\right| \geqslant|m(0)|-\left|m^{\prime}(0)\right| \tau>|m(0)|-\left|m^{\prime}(0)\right| \tau_{0} \geqslant \frac{|m(0)|}{2}>0 .
$$

For each $i \in\{1, \ldots, n\}$, the following recursive deduction can be made. Let $u_{0}, \ldots, u_{i-1} \in \mathrm{L}^{2}(\Omega)$ and $K_{1}, \ldots, K_{i-1} \in \mathbb{R}$ be given. Then, (DMPi] implies the existence of a unique $K_{i} \in \mathbb{R}$ such that

$$
K_{i}\left[m(0)+m^{\prime}(0) \tau\right]=\left(f\left(u_{i-1}\right), 1\right)+\left(F_{i}^{\prime}, 1\right)-\left(g_{i}^{\prime}, 1\right)_{\Gamma}-\sum_{k=1}^{i-1} K_{k} m_{i-k}^{\prime} \tau-m_{i}^{\prime \prime} .
$$

The operator $A$ defined in (7) is strict monotone because for all $u, v \in \mathrm{H}^{1}(\Omega)$ holds that

$$
\langle A(u)-A(v), u-v)\rangle=\frac{1}{\tau}\left(\beta^{-1}(u)-\beta^{-1}(v), u-v\right)+(\nabla u-\nabla v, \nabla u-\nabla v) \geqslant \min \left\{\frac{1}{\beta_{1} \tau}, 1\right\}\|u-v\|_{\mathrm{H}^{1}(\Omega)}^{2} .
$$

From $A(0)=0$, it follows that $A$ is coercive. The continuity of $\beta^{-1}$ implies the demicontinuity of $A$. Moreover, the functional $\tilde{F}_{i}$ defined in $(8)$ is bounded. Starting from $u_{0} \in \mathrm{L}^{2}(\Omega)$, the operator equation $A\left(v_{i}\right)=\tilde{F}_{i}$ has a unique 
solution $v_{i} \in \mathrm{H}^{1}(\Omega)$ for every $i=1, \ldots, n[35]$. Therefore, for $i=1, \ldots, n, u_{i}=\beta^{-1}\left(v_{i}\right)$ is uniquely determined in $\mathrm{H}^{1}(\Omega)$. Finally, the relation 99 yields

$$
\left|K_{i}\right| \leqslant C\left(1+\sum_{k=1}^{i-1}\left|K_{k}\right| \tau\right)
$$

which is valid for any $i=1, \ldots, n$. An application of the discrete Grönwall lemma [36] gives the uniform bound of $\left|K_{i}\right|$.

\section{A priori estimates}

The a priori estimates proved in this section serve as uniform bounds to prove convergence.

Lemma 5.1. Let the conditions of Theorem 4.1 be satisfied. Then there exist positive constants $C$ such that for any $\tau<\tau_{0}$ holds that

$$
\max _{1 \leqslant j \leqslant n}\left\|u_{j}\right\|^{2}+\sum_{i=1}^{n}\left\|\nabla \beta\left(u_{i}\right)\right\|^{2} \tau \leqslant C
$$

and

$$
\max _{0 \leqslant j \leqslant n}\left\|\beta\left(u_{j}\right)\right\| \leqslant C \quad \text { and } \quad \sum_{i=1}^{n}\left\|u_{i}\right\|_{\mathrm{H}^{1}(\Omega)}^{2} \tau \leqslant C .
$$

Proof. We set $\phi=\beta\left(u_{i}\right) \tau$ in (DPi) and sum up for $i=1, \ldots, j$, we obtain that

$$
\begin{aligned}
\sum_{i=1}^{j}\left(\delta u_{i}, \beta\left(u_{i}\right)\right) & \tau+\sum_{i=1}^{j}\left\|\nabla \beta\left(u_{i}\right)\right\|^{2} \tau \\
= & \sum_{i=1}^{j}\left(\sum_{k=0}^{i-1} f\left(u_{k}\right) \tau, \beta\left(u_{i}\right)\right) \tau+\sum_{i=1}^{j}\left(F_{i}, \beta\left(u_{i}\right)\right) \tau-\sum_{i=1}^{j}\left(g_{i}, \beta\left(u_{i}\right)\right)_{\Gamma} \tau-\sum_{i=1}^{j}\left(\sum_{k=1}^{i} K_{k} u_{i-k} \tau, \beta\left(u_{i}\right)\right) \tau .
\end{aligned}
$$

For the first term on the left-hand side (LHS), we get that

$$
\sum_{i=1}^{j}\left(u_{i}-u_{i-1}, \beta\left(u_{i}\right)\right) \stackrel{6}{\geqslant} \sum_{i=1}^{j}\left(\int_{\Omega} \Phi_{\beta}\left(u_{i}\right)-\int_{\Omega} \Phi_{\beta}\left(u_{i-1}\right)\right)=\int_{\Omega} \Phi_{\beta}\left(u_{j}\right)-\int_{\Omega} \Phi_{\beta}\left(u_{0}\right) .
$$

Moreover,

$$
\int_{\Omega} \Phi_{\beta}\left(u_{j}\right) \stackrel{55}{\frac{5}{2}} \frac{\beta_{0}}{2}\left\|u_{j}\right\|^{2} \quad \text { and } \quad\left|\int_{\Omega} \Phi_{\beta}\left(u_{0}\right)\right| \stackrel{5}{\leqslant} \frac{\beta_{1}}{2}\left\|u_{0}\right\|^{2} \leqslant C .
$$

The first two terms on the right-hand side (RHS) of (10) can be estimated by by the boundedness of $f$ and the Lipschitz continuity of $\beta$ as

$$
\left|\sum_{i=1}^{j}\left(\sum_{k=0}^{i-1} f\left(u_{k}\right) \tau, \beta\left(u_{i}\right)\right) \tau\right| \leqslant C+C \sum_{i=1}^{j}\left\|u_{i}\right\|^{2} \tau \quad \text { and } \quad\left|\sum_{i=1}^{j}\left(F_{i}, \beta\left(u_{i}\right)\right) \tau\right| \leqslant C+C \sum_{i=1}^{j}\left\|u_{i}\right\|^{2} \tau .
$$

The third therm can be estimated by using the Nečas inequality [37], i.e.

$$
\|z\|_{\Gamma}^{2} \leqslant \varepsilon\|\nabla z\|^{2}+C_{\varepsilon}\|z\|^{2}, \quad \forall z \in \mathrm{H}^{1}(\Omega), \quad 0<\varepsilon<\varepsilon_{0} .
$$

Due to the properties of $\beta$, it holds true that

$$
\left|\sum_{i=1}^{j}\left(g_{i}, \beta\left(u_{i}\right)\right)_{\Gamma} \tau\right| \leqslant C_{\varepsilon}+C_{\varepsilon} \sum_{\substack{i=1 \\ 5}}^{j}\left\|u_{i}\right\|^{2} \tau+\varepsilon \sum_{i=1}^{j}\left\|\nabla \beta\left(u_{i}\right)\right\|^{2} \tau .
$$


By Young's inequality, Hölder's inequality, Theorem 4.1 and $u_{0} \in \mathrm{L}^{2}(\Omega)$, we obtain that

$$
\left|\sum_{i=1}^{j}\left(\sum_{k=1}^{i} K_{k} u_{i-k} \tau, \beta\left(u_{i}\right)\right) \tau\right| \leqslant \frac{1}{2} \sum_{i=1}^{j}\left(\sum_{k=1}^{i} K_{k}^{2} \tau\right)\left(\sum_{k=1}^{i}\left\|u_{i-k}\right\|^{2} \tau\right) \tau+\frac{1}{2} \sum_{i=1}^{j}\left\|\beta\left(u_{i}\right)\right\|^{2} \tau \leqslant C+C \sum_{i=1}^{j}\left\|u_{i}\right\|^{2} \tau .
$$

Collecting the previous estimates and fixing $\varepsilon$ sufficiently small gives that

$$
\left\|u_{j}\right\|^{2}+\sum_{i=1}^{j}\left\|\nabla \beta\left(u_{i}\right)\right\|^{2} \tau \leqslant C+C \sum_{i=1}^{j}\left\|u_{i}\right\|^{2} \tau
$$

An application of the discrete Grönwall lemma concludes the proof.

Lemma 5.2. Let the conditions of Theorem 4.1 be satisfied and $u_{0} \in \mathrm{H}^{1}(\Omega)$. Then there exist positive constants $C$ such that for any $\tau<\tau_{0}$ holds that

$$
\sum_{i=1}^{n}\left\|\delta u_{i}\right\|^{2} \tau+\max _{1 \leqslant j \leqslant n}\left\|\nabla \beta\left(u_{j}\right)\right\|^{2}+\sum_{i=1}^{j}\left\|\nabla \beta\left(u_{i}\right)-\nabla \beta\left(u_{i-1}\right)\right\|^{2} \leqslant C
$$

and

$$
\max _{1 \leqslant j \leqslant n}\left\|u_{j}\right\|_{\mathrm{H}^{1}(\Omega)} \leqslant C \quad \text { and } \quad \sum_{i=1}^{j}\left\|\nabla u_{i}-\nabla u_{i-1}\right\|^{2} \leqslant C .
$$

Proof. First, we put $\phi=\beta\left(u_{i}\right)-\beta\left(u_{i-1}\right)$ in $(\mathrm{DP} i)$ and sum up for $i=1, \ldots, j$, we get that

$$
\begin{aligned}
\sum_{i=1}^{j}\left(\delta u_{i}, \beta\left(u_{i}\right)-\right. & \left.\beta\left(u_{i-1}\right)\right)+\sum_{i=1}^{j}\left(\nabla \beta\left(u_{i}\right), \nabla \beta\left(u_{i}\right)-\nabla \beta\left(u_{i-1}\right)\right)=\sum_{i=1}^{j}\left(\sum_{k=0}^{i-1} f\left(u_{k}\right) \tau, \beta\left(u_{i}\right)-\beta\left(u_{i-1}\right)\right) \\
& +\sum_{i=1}^{j}\left(F_{i}, \beta\left(u_{i}\right)-\beta\left(u_{i-1}\right)\right)-\sum_{i=1}^{j}\left(g_{i}, \beta\left(u_{i}\right)-\beta\left(u_{i-1}\right)\right)_{\Gamma}-\sum_{i=1}^{j}\left(\sum_{k=1}^{i} K_{k} u_{i-k} \tau, \beta\left(u_{i}\right)-\beta\left(u_{i-1}\right)\right) .
\end{aligned}
$$

The strict monotonicity of $\beta$ implies that

$$
\sum_{i=1}^{j}\left(\delta u_{i}, \beta\left(u_{i}\right)-\beta\left(u_{i-1}\right)\right) \geqslant \beta_{0} \sum_{i=1}^{j}\left\|\delta u_{i}\right\|^{2} \tau .
$$

For the second term on the LHS of [12, we apply Abel's lemma [38], which states that

$$
2 \sum_{i=1}^{j} a_{i}\left(a_{i}-a_{i-1}\right)=a_{j}^{2}-a_{0}^{2}+\sum_{i=1}^{j}\left(a_{i}-a_{i-1}\right)^{2} .
$$

Hence (consider $\nabla \beta\left(u_{i}\right)$ instead of $a_{i}$ and integrate over $\Omega$ ),

$$
\sum_{i=1}^{j}\left(\nabla \beta\left(u_{i}\right), \nabla \beta\left(u_{i}\right)-\nabla \beta\left(u_{i-1}\right)\right)=\frac{1}{2}\left\|\nabla \beta\left(u_{j}\right)\right\|^{2}-\frac{1}{2}\left\|\nabla \beta\left(u_{0}\right)\right\|^{2}+\frac{1}{2} \sum_{i=1}^{j}\left\|\nabla \beta\left(u_{i}\right)-\nabla \beta\left(u_{i-1}\right)\right\|^{2} .
$$

On all the first term on the RHS of [12, we apply the following summation by parts formula: for any real sequences $\left\{z_{i}\right\}_{i=0}^{\infty}$ and $\left\{w_{i}\right\}_{i=0}^{\infty}$ holds that

$$
\sum_{i=1}^{j} z_{i}\left(w_{i}-w_{i-1}\right)=z_{j} w_{j}-z_{0} w_{0}-\sum_{i=1}^{j}\left(z_{i}-z_{i-1}\right) w_{i-1}
$$


Therefore,

$$
\begin{aligned}
\sum_{i=1}^{j}\left(\sum_{k=0}^{i-1} f\left(u_{k}\right) \tau, \beta\left(u_{i}\right)-\beta\left(u_{i-1}\right)\right) & =\left(\sum_{k=0}^{j-1} f\left(u_{k}\right) \tau, \beta\left(u_{j}\right)\right)-\sum_{i=1}^{j}\left(f\left(u_{i-1}\right), \beta\left(u_{i-1}\right)\right) \tau \\
\sum_{i=1}^{j}\left(F_{i}, \beta\left(u_{i}\right)-\beta\left(u_{i-1}\right)\right) & =\left(F_{j}, \beta\left(u_{j}\right)\right)-\left(F_{0}, \beta\left(u_{0}\right)\right)-\sum_{i=1}^{j}\left(\delta F_{i}, \beta\left(u_{i-1}\right)\right) \tau \\
\sum_{i=1}^{j}\left(g_{i}, \beta\left(u_{i}\right)-\beta\left(u_{i-1}\right)\right)_{\Gamma} & =\left(g_{j}, \beta\left(u_{j}\right)\right)_{\Gamma}-\left(g_{0}, \beta\left(u_{0}\right)\right)_{\Gamma}-\sum_{i=1}^{j}\left(\delta g_{i}, \beta\left(u_{i-1}\right)\right)_{\Gamma} \tau \\
\sum_{i=1}^{j}\left(\sum_{k=1}^{i} K_{k} u_{i-k} \tau, \beta\left(u_{i}\right)-\beta\left(u_{i-1}\right)\right) & =\left(\sum_{k=1}^{j} K_{k} u_{i-k} \tau, \beta\left(u_{j}\right)\right)-\sum_{i=1}^{j}\left(K_{i} u_{0}+\sum_{k=1}^{i-1} K_{k} \delta u_{i-k} \tau, \beta\left(u_{i-1}\right)\right) \tau .
\end{aligned}
$$

Using Lemma 5.1, it is clear that

$$
\left|\sum_{i=1}^{j}\left(\sum_{k=0}^{i-1} f\left(u_{k}\right) \tau, \beta\left(u_{i}\right)-\beta\left(u_{i-1}\right)\right)\right| \leqslant C \quad \text { and } \quad\left|\sum_{i=1}^{j}\left(F_{i}, \beta\left(u_{i}\right)-\beta\left(u_{i-1}\right)\right)\right| \leqslant C .
$$

Due to Nečas inequality (11), Lemma 5.1 and the assumption $u_{0} \in \mathrm{H}^{1}(\Omega)$, we readily obtain that

$$
\left|\sum_{i=1}^{j}\left(g_{i}, \beta\left(u_{i}\right)-\beta\left(u_{i-1}\right)\right)_{\Gamma}\right| \leqslant C_{\varepsilon}+\varepsilon\left\|\nabla \beta\left(u_{j}\right)\right\|^{2} .
$$

For the last term on the RHS of (12), it is clear by Lemma 4.1. Lemma 5.1 and Hölder's inequality that

$$
\begin{aligned}
\left|\sum_{i=1}^{j}\left(\sum_{k=1}^{i} K_{k} u_{i-k} \tau, \beta\left(u_{i}\right)-\beta\left(u_{i-1}\right)\right)\right| & \leqslant C+C \sum_{i=1}^{j}\left\|\sum_{k=1}^{i-1} K_{k} \delta u_{i-k} \tau\right\|^{2} \tau \\
& \leqslant C+C \sum_{i=1}^{j}\left(\sum_{k=1}^{i-1} K_{k}^{2} \tau\right)\left(\sum_{k=1}^{i-1}\left\|\delta u_{i-k}\right\|^{2} \tau\right) \tau \\
& \leqslant C+C \sum_{i=1}^{j}\left(\sum_{k=1}^{i}\left\|\delta u_{k}\right\|^{2} \tau\right) \tau .
\end{aligned}
$$

Combining the previous estimates and fixing $\varepsilon$ sufficiently small gives that

$$
\sum_{i=1}^{j}\left\|\delta u_{i}\right\|^{2} \tau+\left\|\nabla \beta\left(u_{j}\right)\right\|^{2}+\sum_{i=1}^{j}\left\|\nabla \beta\left(u_{i}\right)-\nabla \beta\left(u_{i-1}\right)\right\|^{2} \leqslant C+C \sum_{i=1}^{j}\left(\sum_{k=1}^{i}\left\|\delta u_{k}\right\|^{2} \tau\right) \tau .
$$

An application of the discrete Grönwall lemma concludes the proof.

In the following lemma, the PDE in (1) needs to be satisfied at $t=0$. Hence, the following compatibility conditions are defined:

$$
\delta u_{0}:=\partial_{t} u(0):=F_{0}+\nabla \cdot\left(\nabla \beta\left(u_{0}\right)\right) \in \mathrm{L}^{2}(\Omega) \quad \text { if } \quad \nabla \beta\left(u_{0}\right) \in \mathbf{H}(\operatorname{div} ; \Omega)
$$

and

$$
u_{-1}:=u_{0}-\tau \delta u_{0} \in \mathrm{L}^{2}(\Omega) .
$$

Now, the discrete measured problem (DMPi $)$ is valid at $t=0$. It holds that

$$
m_{0}^{\prime \prime}+K_{0} m_{0}=\left(f\left(u_{-1}\right), 1\right)+\left(F_{0}^{\prime}, 1\right)-\left(g_{0}^{\prime}, 1\right)_{\Gamma},
$$

which defines $K_{0}$. 
Lemma 5.3. Let the assumptions of Lemma 5.2 be fulfilled. Moreover, assume that $\nabla \beta\left(u_{0}\right) \in \mathbf{H}(\mathbf{d i v} ; \Omega), g \in$ $\mathrm{C}^{2}\left([0, T], \mathrm{L}^{2}(\Gamma)\right), F \in \mathrm{C}^{2}\left([0, T], \mathrm{L}^{2}(\Omega)\right), m \in \mathrm{C}^{3}([0, T])$ and $f: \mathbb{R} \rightarrow \mathbb{R}$ is global Lipschitz continuous. Then, there exist positive constants $C$ and $\tau_{0}$ such that for all $\tau<\tau_{0}$ holds that

$$
\sum_{i=1}^{n}\left|\delta K_{i}\right|^{2} \tau \leqslant C
$$

Proof. First, lets subtract (DMP0 from (DMPi for $i=1$ and divide the result by $\tau$. We obtain

$$
\delta K_{1} m_{0}=\left(f\left(u_{0}\right)-f\left(u_{-1}\right), 1\right)+\left(\delta F_{1}^{\prime}, 1\right)-\left(\delta g_{1}^{\prime}, 1\right)_{\Gamma}-\delta m_{1}^{\prime \prime}-K_{1} m_{0}^{\prime} .
$$

By the mean value theorem and the Lipschitz continuity of $f$, we get for $\tau<\tau_{0}$ holds that

$$
\left|\delta K_{1}\right| \leqslant C\left\|\delta u_{0}\right\| \tau+C \leqslant C,
$$

as $m(0) \neq 0$. Next, we apply the $\delta$-operator to (DMPi for $2 \leqslant i \leqslant n$. This gives us

$$
\delta K_{i} m(0)=\left(\delta f\left(u_{i-1}\right), 1\right)+\left(\delta F_{i}^{\prime}, 1\right)-\left(\delta g_{i}^{\prime}, 1\right)_{\Gamma}-\delta m_{i}^{\prime \prime}-K_{i} m_{0}^{\prime}-\sum_{k=1}^{i-1} K_{k} \delta m_{i-k}^{\prime} \tau .
$$

Theorem 4.1 and the Lipschitz continuity of $f$ lead to

$$
\left|\delta K_{i}\right| \leqslant C\left(1+\left\|\delta u_{i-1}\right\|\right) \quad \text { for } \quad 2 \leqslant i \leqslant n .
$$

Therefore, for $\tau<\tau_{0}$, Lemma 5.2 implies that

$$
\sum_{i=1}^{n}\left|\delta K_{i}\right|^{2} \tau=\left|\delta K_{1}\right|^{2} \tau+\sum_{i=2}^{n}\left|\delta K_{i}\right|^{2} \tau \leqslant C\left(1+\sum_{i=2}^{n}\left\|\delta u_{i-1}\right\|^{2} \tau\right) \leqslant C .
$$

\section{Convergence and existence of a solution}

Let us introduce the following piecewise linear function in time

$$
u_{n}:[0, T] \rightarrow \mathrm{L}^{2}(\Omega): t \mapsto\left\{\begin{array}{ll}
u_{0} & t=0 \\
u_{i-1}+\left(t-t_{i-1}\right) \delta u_{i} & t \in\left(t_{i-1}, t_{i}\right]
\end{array}, \quad 1 \leqslant i \leqslant n,\right.
$$

and a step function

$$
\bar{u}_{n}:[0, T] \rightarrow \mathrm{L}^{2}(\Omega): t \mapsto\left\{\begin{array}{ll}
u_{0} & t=0 \\
u_{i} & t \in\left(t_{i-1}, t_{i}\right]
\end{array}, \quad 1 \leqslant i \leqslant n .\right.
$$

Similarly, define $\bar{K}_{n}, \bar{F}_{n},{\overline{F^{\prime}}}_{n}, \bar{g}_{n}, \overline{g^{\prime}}{ }_{n},{\overline{m^{\prime}}}_{n}$ and $\overline{m^{\prime \prime}}{ }_{n}$. Using these so-called Rothe's functions, (DPi) and (DMPi) can be rewritten on the whole time frame as]

$$
\left(\partial_{t} u_{n}(t), \phi\right)+\left(\nabla \beta\left(\bar{u}_{n}(t)\right), \nabla \phi\right)+\left(\sum_{k=1}^{[t]_{\tau}} \bar{K}_{n}\left(t_{k}\right) \bar{u}_{n}\left(t-t_{k}\right) \tau, \phi\right)=\left(\sum_{k=0}^{\lfloor t]_{\tau}} f\left(\bar{u}_{n}\left(t_{k}\right)\right) \tau, \phi\right)+\left(\bar{F}_{n}(t), \phi\right)-\left(\bar{g}_{n}(t), \phi\right)_{\Gamma}
$$

and

$$
{\overline{m^{\prime \prime}}}_{n}(t)+\bar{K}_{n}(t) m(0)+\sum_{k=1}^{\left\lceil t \tau_{\tau}\right.} \bar{K}_{n}\left(t_{k}\right) \bar{m}_{n}^{\prime}\left(t-t_{k}\right) \tau=\left(f\left(\bar{u}_{n}(t-\tau)\right), 1\right)+\left({\overline{F^{\prime}}}_{n}(t), 1\right)-\left({\overline{g^{\prime}}}_{n}(t), 1\right)_{\Gamma}
$$

This puts us in a position to prove the existence of a weak solution to $(\mathrm{P})$ and $(\mathrm{MP})$.

${ }^{1}\lceil t\rceil_{\tau}=i$ and $\lfloor t\rfloor_{\tau}=i-1$ when $t \in\left(t_{i-1}, t_{i}\right]$ 
Theorem 6.1 (Existence). Suppose the conditions of Lemma 5.2 are fulfilled. Moreover, let $f: \mathbb{R} \rightarrow \mathbb{R}$ be global Lipschitz continuous. Then there exists a weak solution $\langle K, u\rangle$ to the problem $(\mathbb{P})-(\overline{M P})$, where $K \in \mathrm{L}^{2}(0, T)$ and $u \in \mathrm{C}\left([0, T], \mathrm{L}^{2}(\Omega)\right) \cap \mathrm{L}^{2}\left((0, T), \mathrm{H}^{1}(\Omega)\right)$ with $\partial_{t} u \in \mathrm{L}^{2}\left((0, T), \mathrm{L}^{2}(\Omega)\right)$.

Proof. From Lemmas 5.1 and 5.2 , we have for all $n>0$ that

$$
\max _{t \in[0, T]}\left\|\bar{u}_{n}(t)\right\|_{\mathrm{H}^{1}(\Omega)} \leqslant C \quad \text { for all } \quad t \in[0, T], \quad \int_{0}^{T}\left\|\partial_{t} u_{n}(t)\right\|^{2} \mathrm{~d} t \leqslant C .
$$

The Rellich-Kondrachov theorem [39, §5.8.1] implies the compact embedding

$$
\mathrm{H}^{1}(\Omega) \hookrightarrow \hookrightarrow \mathrm{L}^{2}(\Omega) .
$$

Then, the conditions of [34, Lemma 1.3.13] are satisfied. This implies the existence of an element

$$
u \in \mathrm{C}\left([0, T], \mathrm{L}^{2}(\Omega)\right) \cap \mathrm{L}^{\infty}\left((0, T), \mathrm{H}^{1}(\Omega)\right),
$$

and a subsequence $\left(u_{n_{l}}\right)_{k \in \mathbb{N}}$ of $\left(u_{n}\right)_{n \in \mathbb{N}}$ such that

$$
\left\{\begin{array}{lll}
u_{n_{l}} \rightarrow u, & \text { in } & \mathrm{C}\left([0, T], \mathrm{L}^{2}(\Omega)\right), \\
u_{n_{l}}(t) \rightarrow u(t), & \text { in } & \mathrm{H}^{1}(\Omega) \text { for all } t \in[0, T], \\
\bar{u}_{n_{l}}(t)-u(t), & \text { in } & \mathrm{H}^{1}(\Omega) \text { for all } t \in[0, T], \\
\partial_{t} u_{n_{l}} \rightarrow \partial_{t} u, & \text { in } & \mathrm{L}^{2}\left((0, T), \mathrm{L}^{2}(\Omega)\right),
\end{array}\right.
$$

which we denote again by $u_{n}$ for ease of reading. Note that $u_{n}(0)-\bar{u}_{n}(0)=0$. For all $t \in\left(t_{i-1}, t_{i}\right]$ with $1 \leqslant i \leqslant n$, we have that

$$
\left|u_{n}(t)-\bar{u}_{n}(t)\right|=\left|u_{i-1}+\left(t-t_{i-1}\right) \delta u_{i}-u_{i}\right|=\left|\left(t-t_{i-1}-\tau\right) \delta u_{i}\right|=\left|\left(t-t_{i}\right) \delta u_{i}\right| \leqslant \tau\left|\delta u_{i}\right| .
$$

Employing Lemma 5.2 gives

$$
\lim _{n \rightarrow \infty}\left\|u_{n}-\bar{u}_{n}\right\|_{\mathrm{L}^{2}\left((0, T), \mathrm{L}^{2}(\Omega)\right)}^{2} \leqslant \lim _{n \rightarrow \infty} \tau^{2} \sum_{i=1}^{n}\left\|\delta u_{i}\right\|^{2} \tau \leqslant \lim _{n \rightarrow \infty} \frac{C}{n^{2}}=0,
$$

such that $u_{n}$ and $\bar{u}_{n}$ have the same limit in $\mathrm{L}^{2}\left((0, T), \mathrm{L}^{2}(\Omega)\right)$, i.e.

$$
\bar{u}_{n} \rightarrow u \quad \text { in } \quad \mathrm{L}^{2}\left((0, T), \mathrm{L}^{2}(\Omega)\right) .
$$

By [40, p. 88], the exists a subsequence $\left\{\bar{u}_{n}\right\}$ of $\left\{\bar{u}_{n}\right\}$ such that

$$
\bar{u}_{n} \rightarrow u, \quad \text { a.e. in } \quad(0, T) \times \Omega .
$$

Therefore, by the Lipschitz continuity of $\beta$, we get that

$$
\beta\left(\bar{u}_{n}\right) \rightarrow \beta(u) \quad \text { in } \mathrm{L}^{2}\left((0, T), \mathrm{L}^{2}(\Omega)\right) .
$$

From Lemma 5.2 follows that

$$
\int_{0}^{T}\left\|\beta\left(\bar{u}_{n}(t)\right)\right\|_{\mathrm{H}^{1}(\Omega)}^{2} \mathrm{~d} t \leqslant C .
$$

By the reflexivity of the space $\mathrm{L}^{2}\left((0, T), \mathrm{H}^{1}(\Omega)\right)$, we get the existence of a subsequence (again denoted with the same index) such that $\beta\left(\bar{u}_{n}\right) \rightarrow z$ in $\mathrm{L}^{2}\left((0, T), \mathrm{H}^{1}(\Omega)\right)$. Due to $[16)$, we obtain that $z=\beta(u)$. Therefore,

$$
\nabla \beta\left(\bar{u}_{n}\right) \rightarrow \nabla \beta(u) \quad \text { in } \quad \mathrm{L}^{2}\left((0, T), \mathrm{L}^{2}(\Omega)\right) .
$$


Using Theorem 4.1 , we have that $\int_{0}^{T}\left|\bar{K}_{n}(t)\right|^{2} \mathrm{~d} t \leqslant C$, which means by the reflexivity of $\mathrm{L}^{2}(0, T)$ that (in the sense of a subsequence being indexed by $n$ as well)

$$
\bar{K}_{n} \rightarrow K \quad \text { in } \mathrm{L}^{2}(0, T) .
$$

It is clear that $\lim _{n \rightarrow \infty}{\overline{m^{\prime}}}_{n}(t)=m^{\prime}(t)$ and $\lim _{n \rightarrow \infty}{\overline{m^{\prime \prime}}}_{n}(t)=m^{\prime \prime}(t)$ in $\mathrm{L}^{2}([0, T]), \lim _{n \rightarrow \infty} \bar{g}_{n}(t)=g(t)$ and $\lim _{n \rightarrow \infty}{\overline{g^{\prime}}}_{n}(t)=g^{\prime}(t)$ in $\mathrm{L}^{2}\left((0, T), \mathrm{L}^{2}(\Gamma)\right)$ and $\lim _{n \rightarrow \infty} \bar{F}_{n}(t)=F(t)$ and $\lim _{n \rightarrow \infty}{\overline{F^{\prime}}}_{n}(t)=F^{\prime}(t)$ in $\mathrm{L}^{2}\left((0, T), \mathrm{L}^{2}(\Omega)\right)$ because $m, F$ and $g$ are prescribed. Now, we integrate $(\mathrm{DP})$ in time over $(0, \eta) \subset[0, T]$ for the resulting subsequence to get

$$
\begin{aligned}
\int_{0}^{\eta}\left(\partial_{t} u_{n}(t), \phi\right) \mathrm{d} t+\int_{0}^{\eta}\left(\nabla \beta\left(\bar{u}_{n}(t)\right), \nabla \phi\right) \mathrm{d} t & +\int_{0}^{\eta}\left(\sum_{k=1}^{\lceil t\rceil_{\tau}} \bar{K}_{n}\left(t_{k}\right) \bar{u}_{n}\left(t-t_{k}\right) \tau, \phi\right) \mathrm{d} t \\
& =\int_{0}^{\eta}\left(\sum_{k=0}^{\lfloor t\rfloor_{\tau}} f\left(\bar{u}_{n}\left(t_{k}\right)\right) \tau, \phi\right) \mathrm{d} t+\int_{0}^{\eta}\left(\bar{F}_{n}(t), \phi\right) \mathrm{d} t-\int_{0}^{\eta}\left(\bar{g}_{n}(t), \phi\right)_{\Gamma} \mathrm{d} t .
\end{aligned}
$$

This expression is valid for any $\eta \in[0, T]$. We want to pass the limit $n \rightarrow \infty$ in (19). Using the stability result 14a, we have for $n \rightarrow \infty$ that

$$
\int_{0}^{\eta}\left(\partial_{t} u_{n}, \phi\right) \rightarrow \int_{0}^{\eta}\left(\partial_{t} u, \phi\right), \quad \forall \phi \in \mathrm{H}^{1}(\Omega)
$$

Thanks to (17), we have that

$$
\int_{0}^{\eta}\left(\nabla \beta\left(\bar{u}_{n}(t)\right), \nabla \phi\right) \mathrm{d} t \rightarrow \int_{0}^{\eta}(\nabla \beta(u(t)), \nabla \phi) \mathrm{d} t, \quad \forall \phi \in \mathrm{H}^{1}(\Omega) .
$$

By Theorem 4.1 and Lemma 5.1, it holds for all $t \in[0, T]$ that

$$
\sum_{k=1}^{[t]_{\tau}} \bar{K}_{n}\left(t_{k}\right) \bar{u}_{n}\left(t-t_{k}\right) \tau=\left(\bar{K}_{n} * \bar{u}_{n}\right)(t)+\int_{t}^{\tau\lceil t]_{\tau}} \bar{K}_{n}(s) \bar{u}_{n}(t-s) \mathrm{d} s=\left(\bar{K}_{n} * \bar{u}_{n}\right)(t)+O(\tau)
$$

and

$$
\sum_{k=0}^{\lfloor t\rfloor_{\tau}} f\left(\bar{u}_{n}\left(t_{k}\right)\right) \tau=f\left(u_{0}\right) \tau+\int_{0}^{t} f\left(\bar{u}_{n}(s)\right) \mathrm{d} s-\int_{\tau\lfloor t\rfloor_{\tau}}^{t} f\left(\bar{u}_{n}(s)\right) \mathrm{d} s=\int_{0}^{t} f\left(\bar{u}_{n}(s)\right) \mathrm{d} s+O(\tau) .
$$

Hence, by [15], (18) and the Lipschitz continuity of $f$, we see that

$$
\lim _{n \rightarrow \infty} \int_{0}^{\eta}\left(\sum_{k=1}^{[t\rceil_{\tau}} \bar{K}_{n}\left(t_{k}\right) \bar{u}_{n}\left(t-t_{k}\right) \tau, \phi\right) \mathrm{d} t=\lim _{n \rightarrow \infty} \int_{0}^{\eta} \sum_{k=1}^{[t\rceil_{\tau}} \bar{K}_{n}\left(t_{k}\right)\left(\bar{u}_{n}\left(t-t_{k}\right), \phi\right) \tau=\int_{0}^{\eta}((K * u)(t), \phi) \mathrm{d} t
$$

and

$$
\lim _{n \rightarrow \infty} \int_{0}^{\eta}\left(\sum_{k=0}^{\lfloor t\rfloor_{\tau}} f\left(\bar{u}_{n}\left(t_{k}\right)\right) \tau, \phi\right) \mathrm{d} t=\int_{0}^{\eta}\left(\int_{0}^{t} f(u(s)) \mathrm{d} s, \phi\right) \mathrm{d} s .
$$

Now, taking the limit $n \rightarrow \infty$ in 19) results in

$$
\begin{aligned}
\int_{0}^{\eta}\left(\partial_{t} u(t), \phi\right) \mathrm{d} t+\int_{0}^{\eta}(\nabla \beta(u(t)), \nabla \phi) \mathrm{d} t+ & \int_{0}^{\eta}((K * u)(t), \phi) \mathrm{d} t \\
& =\int_{0}^{\eta}\left(\int_{0}^{t} f(u(s)) \mathrm{d} s, \phi\right) \mathrm{d} t+\int_{0}^{\eta}(F(t), \phi) \mathrm{d} t-\int_{0}^{\eta}(g(t), \phi)_{\Gamma} \mathrm{d} t .
\end{aligned}
$$


Taking the derivative with respect to $\eta$, we arrive at $(\mathrm{P})$. In the same way as before, we integrate $(\overline{\mathrm{DMP}})$ in time and pass the limit for $n \rightarrow \infty$. This follows the same line as passing the limit in (19), therefore we skip the details. Note that

$$
\lim _{n \rightarrow \infty} \int_{0}^{T}\left\|\bar{u}_{n}(t-\tau)-\bar{u}_{n}(t)\right\|^{2} \mathrm{~d} t=0
$$

Finally, we differentiate the result with respect to time and arrive at $(\mathrm{MP})$.

In the previous theorem, to prove the existence of a weak solution to $(\mathrm{P})-(\overline{\mathrm{MP}})$, only the weak convergence of $\bar{K}_{n}$ to $K$ in $\mathrm{L}^{2}(0, T)$ is used. However, assume that the conditions of Lemma 5.3 are satisfied. Then, from this lemma, it is clear that

$$
\max _{t \in[0, T]}\left|K_{n}(t)\right| \leqslant C \quad \text { for all } t \text { in }[0, T] \quad \text { and } \quad \int_{0}^{T}\left|\partial_{t} K_{n}(t)\right|^{2} \mathrm{~d} t \leqslant C .
$$

From this follows that $\bar{K}_{n}$ and $K_{n}$ have the same limit in $\mathrm{L}^{2}(0, T)$. Moreover, by the Arzelà-Ascoli theorem [41, Theorem 11.28], there exists a subsequence $\left\{K_{n}\right\}$ (which we denote by the same symbol again) that converges uniformly on $[0, T]$ to $K$, i.e. $K \in \mathrm{C}([0, T])$. The reflexivity of the space $\mathrm{L}^{2}(0, T)$ implies that $\partial_{t} K_{n} \rightarrow \partial_{t} K$ in $\mathrm{L}^{2}(0, T)$.

Corollary 6.1. Suppose the conditions of Lemma 5.3 are fulfilled. Then there exists a weak solution $\langle K, u\rangle$ to the problem $(\mathrm{P})-(\mathrm{MP})$, where $K \in \mathrm{C}([0, T])$ with $K^{\prime} \in \mathrm{L}^{2}(0, T)$ and $u \in \mathrm{C}\left([0, T], \mathrm{L}^{2}(\Omega)\right) \cap \mathrm{L}^{2}\left((0, T), \mathrm{H}^{1}(\Omega)\right)$ with $\partial_{t} u \in \mathrm{L}^{2}\left((0, T), \mathrm{L}^{2}(\Omega)\right)$.

Now, it is possible to establish the uniqueness of a solution to $(\mathrm{P})$ - $\mathrm{MP}$.

Theorem 6.2 (Uniqueness). Let the assumptions of Lemma 6.1] be satisfied. Then the problem (P)-(MP) has at most one solution $\langle K, u\rangle \in \mathrm{L}^{2}(0, T) \times\left[\mathrm{C}\left([0, T], \mathrm{L}^{2}(\Omega)\right) \cap \mathrm{L}^{2}\left((0, T), \mathrm{H}^{1}(\Omega)\right)\right]$ with $\partial_{t} u \in \mathrm{L}^{2}\left((0, T), \mathrm{L}^{2}(\Omega)\right)$.

Proof. First, we integrate $(\mathbb{P})$ over the time variable $t \in(0, \xi) \subset(0, T)$. We obtain that

$$
\begin{aligned}
\left(u(\xi)-u_{0}, \phi\right)+\left(\int_{0}^{\xi} \nabla \beta(u(t)) \mathrm{d} t, \nabla \phi\right) & +\left(\int_{0}^{\xi}(K * u)(t) \mathrm{d} t, \phi\right) \\
& =\left(\int_{0}^{\xi} \int_{0}^{t} f(u(s)) \mathrm{d} s \mathrm{~d} t, \phi\right)+\left(\int_{0}^{\xi} F(t) \mathrm{d} t, \phi\right)-\left(\int_{0}^{\xi} g(t) \mathrm{d} t, \phi\right)_{\Gamma}
\end{aligned}
$$

Suppose that there are two solutions $\left\langle K_{1}, u_{1}\right\rangle$ and $\left\langle K_{2}, u_{2}\right\rangle$ solving $(\mathrm{P})$ - $(\overline{\mathrm{MP}})$. Denote the difference of the solutions by $K=K_{1}-K_{2}$ and $u=u_{1}-u_{2}$. Subtracting the integrated variational formulation 22) for the different solution gives that

$$
\begin{array}{r}
(u(\xi), \phi)+\left(\int_{0}^{\xi}\left[\nabla \beta\left(u_{1}(t)\right)-\nabla \beta\left(u_{2}(t)\right)\right] \mathrm{d} t, \nabla \phi\right)+\left(\int_{0}^{\xi}\left(K_{1} * u\right)(t) \mathrm{d} t, \phi\right)+\left(\int_{0}^{\xi}\left(K * u_{2}\right)(t) \mathrm{d} t, \phi\right) \\
=\left(\int_{0}^{\xi} \int_{0}^{t}\left[f\left(u_{1}(s)\right)-f\left(u_{2}(s)\right)\right] \mathrm{d} s \mathrm{~d} t, \phi\right) .
\end{array}
$$

Now, we put $\phi=\beta\left(u_{1}(\xi)\right)-\beta\left(u_{2}(\xi)\right)$ and integrate again over the time variable $\xi \in(0, \eta) \subset(0, T)$. This gives

$$
\begin{array}{r}
\int_{0}^{\eta}\left(u(\xi), \beta\left(u_{1}(\xi)\right)-\beta\left(u_{2}(\xi)\right)\right) \mathrm{d} \xi+\int_{0}^{\eta}\left(\int_{0}^{\xi}\left[\nabla \beta\left(u_{1}(t)\right)-\nabla \beta\left(u_{2}(t)\right)\right] \mathrm{d} t, \nabla \beta\left(u_{1}(\xi)\right)-\nabla \beta\left(u_{2}(\xi)\right)\right) \mathrm{d} \xi \\
+\int_{0}^{\eta}\left(\int_{0}^{\xi}\left(K_{1} * u\right)(t) \mathrm{d} t, \beta\left(u_{1}(\xi)\right)-\beta\left(u_{2}(\xi)\right)\right) \mathrm{d} \xi+\int_{0}^{\eta}\left(\int_{0}^{\xi}\left(K * u_{2}\right)(t) \mathrm{d} t, \beta\left(u_{1}(\xi)\right)-\beta\left(u_{2}(\xi)\right)\right) \mathrm{d} \xi \\
=\int_{0}^{\eta}\left(\int_{0}^{\xi} \int_{0}^{t}\left[f\left(u_{1}(s)\right)-f\left(u_{2}(s)\right)\right] \mathrm{d} s \mathrm{~d} t, \beta\left(u_{1}(\xi)\right)-\beta\left(u_{2}(\xi)\right)\right) \mathrm{d} \xi
\end{array}
$$


Subtracting (MP) for the different solutions gives

$$
K(t) m(0)+\left(K * m^{\prime}\right)(t)=\left(f\left(u_{1}(t)\right)-f\left(u_{2}(t)\right), 1\right) .
$$

By the Lipschitz continuity of $f$ and Grönwall's lemma, it follows easily that

$$
|K(t)| \leqslant C\|u(t)\|, \quad t \in[0, T] .
$$

Now, we are able to estimate each term of 23]. The strict monotonicity of $\beta$ implies that

$$
\int_{0}^{\eta}\left(u(\xi), \beta\left(u_{1}(\xi)\right)-\beta\left(u_{2}(\xi)\right)\right) \mathrm{d} \xi \geqslant \beta_{0} \int_{0}^{\eta}\|u(\xi)\|^{2} \mathrm{~d} \xi
$$

For the second term on the LHS, we get by the strict monotonicity of $\beta$ and integration by parts that

$$
\int_{0}^{\eta}\left(\int_{0}^{\xi}\left[\nabla \beta\left(u_{1}(t)\right)-\nabla \beta\left(u_{2}(t)\right)\right] \mathrm{d} t, \nabla \beta\left(u_{1}(\xi)\right)-\nabla \beta\left(u_{2}(\xi)\right)\right) \mathrm{d} \xi=\frac{1}{2}\left\|\int_{0}^{\eta}\left[\nabla \beta\left(u_{1}(\xi)\right)-\nabla \beta\left(u_{2}(\xi)\right)\right] \mathrm{d} \xi\right\|^{2} \geqslant 0 .
$$

By Young's, Hölder's and Jensen's inequality, the Lipschitz continuity of $\beta$ and $K_{1} \in \mathrm{L}^{2}(0, T)$, it follows for the third term on the LHS that

$$
\left|\int_{0}^{\eta}\left(\int_{0}^{\xi}\left(K_{1} * u\right)(t) \mathrm{d} t, \beta\left(u_{1}(\xi)\right)-\beta\left(u_{2}(\xi)\right)\right) \mathrm{d} \xi\right| \leqslant C_{\varepsilon} \int_{0}^{\eta} \int_{0}^{\xi}\|u(t)\|^{2} \mathrm{~d} t \mathrm{~d} \xi+\varepsilon \int_{0}^{\eta}\|u(\xi)\|^{2} \mathrm{~d} \xi .
$$

For the last term on the LHS, we use the fact that $u_{2} \in \mathrm{C}\left([0, T], \mathrm{L}^{2}(\Omega)\right)$ and $[24)$ such that

$$
\left|\int_{0}^{\eta}\left(\int_{0}^{\xi}\left(K * u_{2}\right)(t) \mathrm{d} t, \beta\left(u_{1}(\xi)\right)-\beta\left(u_{2}(\xi)\right)\right) \mathrm{d} \xi\right| \leqslant C_{\varepsilon} \int_{0}^{\eta} \int_{0}^{\xi}\|u(t)\|^{2} \mathrm{~d} t \mathrm{~d} \xi+\varepsilon \int_{0}^{\eta}\|u(\xi)\|^{2} \mathrm{~d} \xi .
$$

The same upper bound can be obtained for the term on the RHS of 23 by the Lipschitz continuity of $f$. Collecting all the estimates above and fixing $\varepsilon$ sufficiently small imply that

$$
\int_{0}^{\eta}\|u(\xi)\|^{2} \mathrm{~d} \xi \leqslant C \int_{0}^{\eta} \int_{0}^{\xi}\|u(t)\|^{2} \mathrm{~d} t \mathrm{~d} \xi
$$

An application of Grönwall's lemma gives that $\int_{0}^{\eta}\|u(\xi)\|^{2} \mathrm{~d} \xi=0$, i.e. $u=0$ or $u_{1}=u_{2}$ a.e. in $\Omega \times[0, T]$. The uniqueness of $K$ in $\mathrm{L}^{2}(0, T)$ follows from (24).

The convergence of Rothe's functions towards the weak solution have been shown for a subsequence $\left\{u_{n}\right\}$ and $\left\{\bar{K}_{n}\right\}$ in Theorem 6.1 However, taking into account the uniqueness of a solution, it is clear that the whole Rothe's sequences $\left\{u_{n}\right\}$ and $\left\{K_{n}\right\}$ converge against the solution.

\section{Error analysis}

For the error analysis, the assumptions of Lemma 5.3 needs to be satisfied. First, the following notations are introduced:

$$
e_{u}=u_{n}-u, \quad e_{\bar{u}}=\bar{u}_{n}-u, \quad e_{\bar{K}}=\bar{K}_{n}-K .
$$

Analogously, $e_{\bar{g}}, e_{\overline{g^{\prime}}}, e_{\bar{F}}, e_{\overline{F^{\prime}}}, e_{\overline{m^{\prime}}}$ and $e_{\overline{m^{\prime \prime}}}$ are defined. Secondly, the following estimates are frequently used

$$
\left\|\bar{u}_{n}(t-\tau)-\bar{u}_{n}(t)\right\|+\left\|\bar{u}_{n}(t)-u_{n}(t)\right\| \leqslant\left\|\partial_{t} u_{n}(t)\right\| \tau, \quad \forall t \in[0, T] .
$$

Note that this inequality is also valid for $t=0$ by compatibility condition (13). Based on the result of Lemma 5.2, this yields

$$
\int_{0}^{T}\left(\left\|\bar{u}_{n}(t-\tau)-\bar{u}_{n}(t)\right\|^{2}+\left\|\bar{u}_{n}(t)-u_{n}(t)\right\|^{2}\right) \mathrm{d} t \leqslant C \tau^{2} .
$$

Finally, the following theorem contains the error estimates. Optimal convergences rates for Rothe's method $(O(\tau))$ are obtained. 
Theorem 7.1. Let the conditions of Lemma 5.3 be fulfilled. Then there exist positive constants $C$ and $\tau_{0}$ such that for all $\tau<\tau_{0}$ holds that

$$
\int_{0}^{T}\left|\bar{K}_{n}(t)-K(t)\right|^{2} \mathrm{~d} t+\int_{0}^{T}\left\|\bar{u}_{n}(t)-u(t)\right\|^{2} \leqslant C \tau^{2} .
$$

Proof. The proof exists out of two steps. First, a bound on $e_{\bar{K}}$ in $\mathrm{L}^{2}(0, T)$ is proved. Afterwards, the error estimates are established. For the first step, we subtract $(\overline{\mathrm{MP}})$ from $(\overline{\mathrm{DMP}})$ and we use an analogue of $(20)$ to get

$$
\begin{aligned}
e_{\overline{m^{\prime \prime}}}(t)+e_{\bar{K}}(t) m_{0}+\int_{t}^{\tau[t]_{\tau}} \bar{K}_{n}(s) \overline{m^{\prime}} n(t-s) \mathrm{d} s+\left(e_{\bar{K}} * \overline{m^{\prime}} n\right)(t)+\left(K * e_{\overline{m^{\prime}}}\right)(t) \\
=\left(f\left(\bar{u}_{n}(t-\tau)\right)-f(u(t)), 1\right)+\left(e_{\overline{F^{\prime}}}(t), 1\right)-\left(e_{\overline{g^{\prime}}}(t), 1\right)_{\Gamma} .
\end{aligned}
$$

By Theorem 4.1 and the mean-value theorem, we obtain that

$$
\left|e_{\overline{m^{\prime \prime}}}(t)\right|+\left|\int_{t}^{\tau[t]_{\tau}} \bar{K}_{n}(s) \overline{m^{\prime}}{ }_{n}(t-s) \mathrm{d} s\right|+\left|\left(K * e_{\overline{m^{\prime}}}\right)(t)\right|+\left|\left(e_{\overline{F^{\prime}}}(t), 1\right)\right|+\left|\left(e_{\overline{g^{\prime}}}(t), 1\right)_{\Gamma}\right| \leqslant C \tau .
$$

For the fourth term on the LHS of $[26$, it is true that

$$
\left|\left(e_{\bar{K}} * \overline{m^{\prime}}\right)(t)\right| \leqslant C \int_{0}^{t}\left|e_{\bar{K}}(s)\right| \mathrm{d} s .
$$

The Lipschitz continuity of $f$ implies for the first term on the RHS of 26] that

$$
\left|\left(f\left(\bar{u}_{n}(t-\tau)\right)-f(u(t)), 1\right)\right| \leqslant C\left\|\bar{u}_{n}(t-\tau)-\bar{u}_{n}(t)\right\|+C\left\|e_{\bar{u}}(t)\right\| .
$$

Gathering all the estimates, we obtain from (26) (after division by $m_{0}$ ) that

$$
\left|e_{\bar{K}}(t)\right| \leqslant C\left(\tau+\left\|\bar{u}_{n}(t-\tau)-\bar{u}_{n}(t)\right\|+\left\|e_{\bar{u}}(t)\right\|+\int_{0}^{t}\left|e_{\bar{K}}(s)\right| \mathrm{d} s\right) .
$$

We take the second power and integrate the result with respect to the time variable. For each $\eta \in[0, T]$ holds that

$$
\int_{0}^{\eta}\left|e_{\bar{K}}(t)\right|^{2} \mathrm{~d} t \leqslant C\left(\tau^{2}+\int_{0}^{\eta}\left\|\bar{u}_{n}(t-\tau)-\bar{u}_{n}(t)\right\|^{2} \mathrm{~d} t+\int_{0}^{\eta}\left\|e_{\bar{u}}(t)\right\|^{2} \mathrm{~d} t+\int_{0}^{\eta} \int_{0}^{t}\left|e_{\bar{K}}(s)\right|^{2} \mathrm{~d} s \mathrm{~d} t\right) .
$$

Applying estimate (25) and Grönwall's argument imply that

$$
\int_{0}^{\eta}\left|e_{\bar{K}}(t)\right|^{2} \mathrm{~d} t \leqslant C\left(\tau^{2}+\int_{0}^{\eta}\left\|e_{\bar{u}}(t)\right\|^{2} \mathrm{~d} t\right)
$$

The second part of the proof starts with the subtraction of $(\bar{P})$ from $(\overline{D P})$. Next, the result is integrated in time over $(0, \eta) \subset(0, T)$. We obtain

$$
\begin{aligned}
\left(u_{n}(\xi)\right. & -u(\xi), \phi)+\left(\int_{0}^{\xi}\left[\nabla \beta\left(\bar{u}_{n}(t)\right)-\nabla \beta(u(t))\right] \mathrm{d} t, \nabla \phi\right)+\left(\int_{0}^{\xi}\left[\sum_{k=1}^{[t]_{\tau}} \bar{K}_{n}\left(t_{k}\right) \bar{u}_{n}\left(t-t_{k}\right) \tau-(K * u)(t)\right] \mathrm{d} t, \phi\right) \\
& =\left(\int_{0}^{\xi}\left[\sum_{k=0}^{[t]_{\tau}} f\left(\bar{u}_{n}\left(t_{k}\right)\right) \tau-\int_{0}^{t} f(u(s)) \mathrm{d} s\right] \mathrm{d} t, \phi\right)+\left(\int_{0}^{\xi}\left(\bar{F}_{n}(t)-F(t)\right) \mathrm{d} t, \phi\right)-\left(\int_{0}^{\xi}\left(\bar{g}_{n}(t)-g(t)\right) \mathrm{d} t, \phi\right)_{\Gamma} .
\end{aligned}
$$


Now, we put $\phi=\beta\left(\bar{u}_{n}(\xi)\right)-\beta(u(\xi))$ and integrate again over the time variable $\xi \in(0, \eta) \subset(0, T)$. This gives

$$
\begin{aligned}
& \int_{0}^{\eta}\left(u_{n}(\xi)-u(\xi), \beta\left(\bar{u}_{n}(\xi)\right)-\beta(u(\xi))\right) \mathrm{d} \xi+\int_{0}^{\eta}\left(\int_{0}^{\xi}\left[\nabla \beta\left(\bar{u}_{n}(t)\right)-\nabla \beta(u(t))\right] \mathrm{d} t, \nabla \beta\left(\bar{u}_{n}(\xi)\right)-\nabla \beta(u(\xi))\right) \mathrm{d} \xi \\
& +\int_{0}^{\eta}\left(\int_{0}^{\xi}\left[\sum_{k=1}^{[t]_{\tau}} \bar{K}_{n}\left(t_{k}\right) \bar{u}_{n}\left(t-t_{k}\right) \tau-(K * u)(t)\right] \mathrm{d} t, \beta\left(\bar{u}_{n}(\xi)\right)-\beta(u(\xi))\right) \mathrm{d} \xi \\
& =\int_{0}^{\eta}\left(\int_{0}^{\xi}\left[\sum_{k=0}^{[t]_{\tau}} f\left(\bar{u}_{n}\left(t_{k}\right)\right) \tau-\int_{0}^{t} f(u(s)) \mathrm{d} s\right] \mathrm{d} t, \beta\left(\bar{u}_{n}(\xi)\right)-\beta(u(\xi))\right) \mathrm{d} \xi \\
& \quad+\int_{0}^{\eta}\left(\int_{0}^{\xi}\left(\bar{F}_{n}(t)-F(t)\right) \mathrm{d} t, \beta\left(\bar{u}_{n}(\xi)\right)-\beta(u(\xi))\right) \mathrm{d} \xi-\int_{0}^{\eta}\left(\int_{0}^{\xi}\left(\bar{g}_{n}(t)-g(t)\right) \mathrm{d} t, \beta\left(\bar{u}_{n}(\xi)\right)-\beta(u(\xi))\right) \mathrm{d} \xi
\end{aligned}
$$

After some rearrangements in the terms and using 20$]$ and 21 , it holds that

$$
\begin{aligned}
& \int_{0}^{\eta}\left(\bar{u}_{n}(\xi)-u(\xi), \beta\left(\bar{u}_{n}(\xi)\right)-\beta(u(\xi))\right) \mathrm{d} \xi+\int_{0}^{\eta}\left(u_{n}(\xi)-\bar{u}_{n}(\xi), \beta\left(\bar{u}_{n}(\xi)\right)-\beta(u(\xi))\right) \mathrm{d} \xi \\
& +\frac{1}{2}\left\|\int_{0}^{\eta}\left[\nabla \beta\left(\bar{u}_{n}(t)\right)-\nabla \beta(u(t))\right] \mathrm{d} t\right\|^{2}+\int_{0}^{\eta}\left(\int_{0}^{\xi}\left(\int_{t}^{\tau t t]_{\tau}} \bar{K}_{n}(s) \bar{u}_{n}(t-s) \mathrm{d} s\right) \mathrm{d} t, \beta\left(\bar{u}_{n}(\xi)\right)-\beta(u(\xi))\right) \mathrm{d} \xi \\
& +\int_{0}^{\eta}\left(\int_{0}^{\xi}\left(e_{\bar{K}} * \bar{u}_{n}\right)(t) \mathrm{d} t, \beta\left(\bar{u}_{n}(\xi)\right)-\beta(u(\xi))\right) \mathrm{d} \xi+\int_{0}^{\eta}\left(\int_{0}^{\xi}\left(K * e_{\bar{u}}\right)(t) \mathrm{d} t, \beta\left(\bar{u}_{n}(\xi)\right)-\beta(u(\xi))\right) \mathrm{d} \xi \\
& =\int_{0}^{\eta}\left(\int_{0}^{\xi}\left[f\left(u_{0}\right) \tau+\int_{t}^{\tau[t]_{\tau}} f\left(\bar{u}_{n}(s)\right) \mathrm{d} s\right] \mathrm{d} t, \beta\left(\bar{u}_{n}(\xi)\right)-\beta(u(\xi))\right) \mathrm{d} \xi \\
& +\int_{0}^{\eta}\left(\int_{0}^{\xi}\left[\int_{0}^{t}\left(f\left(\bar{u}_{n}(s)\right)-f(u(s))\right) \mathrm{d} s\right] \mathrm{d} t, \beta\left(\bar{u}_{n}(\xi)\right)-\beta(u(\xi))\right) \mathrm{d} \xi \\
& \quad+\int_{0}^{\eta}\left(\int_{0}^{\xi} e_{\bar{F}}(t) \mathrm{d} t, \beta\left(\bar{u}_{n}(\xi)\right)-\beta(u(\xi))\right) \mathrm{d} \xi-\int_{0}^{\eta}\left(\int_{0}^{\xi} e_{\bar{g}}(t) \mathrm{d} t, \beta\left(\bar{u}_{n}(\xi)\right)-\beta(u(\xi))\right)_{\Gamma} \mathrm{d} \xi .
\end{aligned}
$$

By the strict monotonicity of $\beta$, we get that

$$
\int_{0}^{\eta}\left(\bar{u}_{n}(\xi)-u(\xi), \beta\left(\bar{u}_{n}(\xi)\right)-\beta(u(\xi))\right) \mathrm{d} \xi \geqslant \beta_{0} \int_{0}^{\eta}\left\|e_{\bar{u}}(\xi)\right\|^{2} \mathrm{~d} \xi .
$$

For the second term on the LHS, by the Cauchy and Young inequalities, the Lipschitz continuity of $\beta$ and [25], we obtain that

$$
\left|\int_{0}^{\eta}\left(u_{n}(\xi)-\bar{u}_{n}(\xi), \beta\left(\bar{u}_{n}(\xi)\right)-\beta(u(\xi))\right) \mathrm{d} \xi\right| \leqslant C_{\varepsilon} \tau^{2}+\varepsilon \int_{0}^{\eta}\left\|e_{\bar{u}}(\xi)\right\|^{2} \mathrm{~d} \xi
$$

Using the Cauchy and Young inequalities, the uniform boundedness of $\bar{K}_{n}$ and $\bar{u}_{n}$ and the Lipschitz continuity of $\beta$, it follows that

$$
\left|\int_{0}^{\eta}\left(\int_{0}^{\xi}\left(\int_{t}^{\tau[t]_{\tau}} \bar{K}_{n}(s) \bar{u}_{n}(t-s) \mathrm{d} s\right) \mathrm{d} t, \beta\left(\bar{u}_{n}(\xi)\right)-\beta(u(\xi))\right) \mathrm{d} \xi\right| \leqslant C_{\varepsilon} \tau^{2}+\varepsilon \int_{0}^{\eta}\left\|e_{\bar{u}}(t)\right\|^{2} \mathrm{~d} t
$$

For the fifth term on the LHS of [28, we use Young's, Hölder's and Jensen's inequality together with the previously 
obtained estimate (27). We obtain that

$$
\begin{aligned}
& \left|\int_{0}^{\eta}\left(\int_{0}^{\xi}\left(e_{\bar{K}} * \bar{u}_{n}\right)(t) \mathrm{d} t, \beta\left(\bar{u}_{n}(\xi)\right)-\beta(u(\xi))\right) \mathrm{d} \xi\right| \\
& \quad \leqslant \quad C_{\varepsilon} \int_{0}^{\eta}\left\|\int_{0}^{\xi}\left(e_{\bar{K}} * \bar{u}_{n}\right)(t) \mathrm{d} t\right\|^{2} \mathrm{~d} \xi+\varepsilon \int_{0}^{\eta}\left\|e_{\bar{u}}(\xi)\right\|^{2} \mathrm{~d} \xi \\
& \quad \leqslant \quad C_{\varepsilon} \int_{0}^{\eta} \int_{0}^{\xi}\left(\int_{0}^{t}\left|e_{\bar{K}}(s)\right|^{2} \mathrm{~d} s\right) \mathrm{d} t \mathrm{~d} \xi+\varepsilon \int_{0}^{\eta}\left\|e_{\bar{u}}(\xi)\right\|^{2} \mathrm{~d} \xi \\
& \quad \stackrel{27}{\leqslant} C_{\varepsilon} \tau^{2}+C_{\varepsilon} \int_{0}^{\eta} \int_{0}^{\xi}\left\|e_{\bar{u}}(s)\right\|^{2} \mathrm{~d} s \mathrm{~d} \xi+\varepsilon \int_{0}^{\eta}\left\|e_{\bar{u}}(\xi)\right\|^{2} \mathrm{~d} \xi .
\end{aligned}
$$

Moreover, in an analogous way, it holds that

$$
\left|\int_{0}^{\eta}\left(\int_{0}^{\xi}\left(K * e_{\bar{u}}\right)(t) \mathrm{d} t, \beta\left(\bar{u}_{n}(\xi)\right)-\beta(u(\xi))\right) \mathrm{d} \xi\right| \leqslant C_{\varepsilon} \int_{0}^{\eta} \int_{0}^{\xi}\left\|e_{\bar{u}}(s)\right\|^{2} \mathrm{~d} s \mathrm{~d} \xi+\varepsilon \int_{0}^{\eta}\left\|e_{\bar{u}}(\xi)\right\|^{2} \mathrm{~d} \xi .
$$

Now, we estimate the terms in the RHS of (28). The first term can be estimated as

$$
\left|\int_{0}^{\eta}\left(\int_{0}^{\xi}\left[f\left(u_{0}\right) \tau+\int_{t}^{\tau[t\rfloor_{\tau}} f\left(\bar{u}_{n}(s)\right) \mathrm{d} s\right] \mathrm{d} t, \beta\left(\bar{u}_{n}(\xi)\right)-\beta(u(\xi))\right) \mathrm{d} \xi\right| \leqslant C_{\varepsilon} \tau^{2}+\varepsilon \int_{0}^{\eta}\left\|e_{\bar{u}}(\xi)\right\|^{2} \mathrm{~d} \xi .
$$

By the Lipschitz continuity of $f$, we calculate that

$$
\begin{aligned}
& \left|\int_{0}^{\eta}\left(\int_{0}^{\xi}\left[\int_{0}^{t}\left(f\left(\bar{u}_{n}(s)\right)-f(u(s))\right) \mathrm{d} s\right] \mathrm{d} t, \beta\left(\bar{u}_{n}(\xi)\right)-\beta(u(\xi))\right) \mathrm{d} \xi\right| \\
& \quad \leqslant C_{\varepsilon} \int_{0}^{\eta} \int_{0}^{\xi}\left\|e_{\bar{u}}(s)\right\|^{2} \mathrm{~d} s \mathrm{~d} \xi+\varepsilon \int_{0}^{\eta}\left\|e_{\bar{u}}(\xi)\right\|^{2} \mathrm{~d} \xi .
\end{aligned}
$$

For the third term on the RHS, we use the mean value theorem such that

$$
\left|\int_{0}^{\eta}\left(\int_{0}^{\xi} e_{\bar{F}}(t) \mathrm{d} t, \beta\left(\bar{u}_{n}(\xi)\right)-\beta(u(\xi))\right) \mathrm{d} \xi\right| \leqslant C_{\varepsilon} \tau^{2}+\varepsilon \int_{0}^{\eta}\left\|e_{\bar{u}}(\xi)\right\|^{2} \mathrm{~d} \xi .
$$

We use the integration by parts formula for the last term on the RHS, i.e.

$$
\begin{aligned}
\int_{0}^{\eta}\left(\int_{0}^{\xi} e_{\bar{g}}(t) \mathrm{d} t, \beta\left(\bar{u}_{n}(\xi)\right)-\beta(u(\xi))\right)_{\Gamma} \mathrm{d} \xi=\left(\int_{0}^{\eta} e_{\bar{g}}(t) \mathrm{d} t, \int_{0}^{\eta} \beta\left(\bar{u}_{n}(t)\right)-\beta(u(t)) \mathrm{d} t\right)_{\Gamma} & \\
& -\int_{0}^{\eta}\left(e_{\bar{g}}(\xi), \int_{0}^{\xi} \beta\left(\bar{u}_{n}(t)\right)-\beta(u(t)) \mathrm{d} t\right)_{\Gamma} \mathrm{d} \xi .
\end{aligned}
$$

We employ Cauchy's and Young's inequalities, the mean value theorem, the trace inequality and the Lipschitz continuity of $\beta$ such that

$$
\begin{aligned}
& \mid \int_{0}^{\eta}\left(\int_{0}^{\xi} e_{\bar{g}}(t) \mathrm{d} t, \beta\left(\bar{u}_{n}(\xi)\right)-\beta(u(\xi))\right) \\
& \Gamma \\
& \mathrm{d} \xi \mid \leqslant C_{\varepsilon} \tau^{2}+\varepsilon \int_{0}^{\eta}\left\|e_{\bar{u}}(t)\right\|^{2} \mathrm{~d} t \\
& +\varepsilon\left\|\int_{0}^{\eta}\left[\nabla \beta\left(\bar{u}_{n}(t)\right)-\nabla \beta(u(t))\right] \mathrm{d} t\right\|^{2}+C \int_{0}^{\eta} \int_{0}^{\xi}\left\|e_{\bar{u}}(s)\right\|^{2} \mathrm{~d} s \mathrm{~d} \xi+C \int_{0}^{\eta}\left\|\int_{0}^{\xi}\left[\nabla \beta\left(\bar{u}_{n}(t)\right)-\nabla \beta(u(t))\right] \mathrm{d} t\right\|^{2} \mathrm{~d} \xi .
\end{aligned}
$$

Collecting all the previous estimates give

$$
\begin{aligned}
\left(\beta_{0}-\varepsilon\right) \int_{0}^{\eta}\left\|e_{\bar{u}}(\xi)\right\|^{2} \mathrm{~d} \xi+ & \left(\frac{1}{2}-\varepsilon\right)\left\|\int_{0}^{\eta}\left[\nabla \beta\left(\bar{u}_{n}(t)\right)-\nabla \beta(u(t))\right] \mathrm{d} t\right\|^{2} \\
& \leqslant C_{\varepsilon} \tau^{2}+C \int_{0}^{\eta} \int_{0}^{\xi}\left\|e_{\bar{u}}(s)\right\|^{2} \mathrm{~d} s \mathrm{~d} \xi+C \int_{0}^{\eta}\left\|\int_{0}^{\xi}\left[\nabla \beta\left(\bar{u}_{n}(t)\right)-\nabla \beta(u(t))\right] \mathrm{d} t\right\|^{2} \mathrm{~d} \xi .
\end{aligned}
$$

Fixing $\varepsilon$ sufficiently small, an application of Grönwall's lemma concludes the proof. 
Corollary 7.1. Let the conditions of Lemma 5.3 be fulfilled. Then there exist positive constants $C$ and $\tau_{0}$ such that for all $\tau<\tau_{0}$ holds that

$$
\int_{0}^{T}\left|K_{n}(t)-K(t)\right|^{2} \mathrm{~d} t \leqslant C \tau^{2}
$$

\section{Numerical Experiments}

In this section, it is assumed that the assumptions of Theorem 7.1 are satisfied. The presented numerical algorithm in the previous sections is summarized in the following pseudo code:

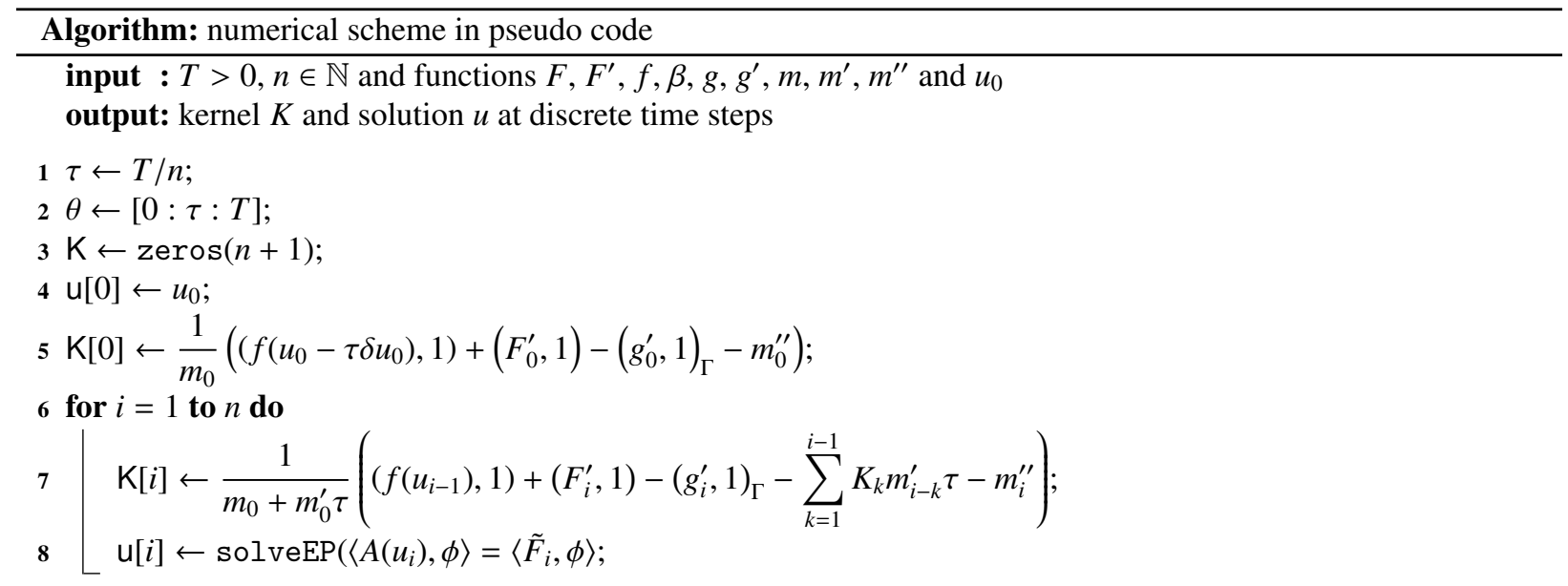

The aim of the simulations is to demonstrated the established error estimate on the convolution kernel $K$ in Corollary 7.1. For these simulations, the finite element library DOLFIN [42, 43] from the FEniCS project [44, 45] is used.

In each experiment, the domain $\Omega=[0,1]$. The number of time discretization interval is chosen to be $n=2^{j}, j=$ $2, \ldots, 8$, such that the time step $\tau$ for the equidistant time partitioning equals respectively $2^{-j} T, j=2, \ldots, 8$. At each time-step, the resulting nonlinear elliptic problems (see Line 8 in the Algorithm) are solved numerically by the finite element method (FEM) using first order (P1-FEM) Lagrange polynomials for the space discretization. At each timestep, the nonlinearity $\nabla \beta\left(u_{i}\right)$ is approximated by $\beta^{\prime}\left(u_{i-1}\right) \nabla u_{i}$. For the space discretization, a fixed uniform mesh of 100 intervals is used. The $\mathrm{L}^{2}$-error between the numerical and exact kernel is approximated by Simpson's rule for the several values of the timestep $\tau$ :

$$
E_{K_{\mathrm{ex}}}(\tau)=\int_{0}^{T}\left|K_{n}(t)-K_{\mathrm{ex}}(t)\right|^{2} \mathrm{~d} t \approx \sum_{i=1}^{n} \frac{\tau}{6}\left[\left(K_{i-1}-K_{\mathrm{ex}}\left(t_{i-1}\right)\right)^{2}+\left(\frac{K_{i-1}+K_{i}}{2}-K_{\mathrm{ex}}\left(\frac{t_{i-1}+t_{i}}{2}\right)\right)^{2}+\left(K_{i}-K_{\mathrm{ex}}\left(t_{i}\right)\right)^{2}\right] .
$$

\subsection{Experiment 1}

The exact solution in the first experiment is prescribed as follows

$$
u_{\mathrm{ex}}(x, t)=\left(1+t+t^{2}\right)\left(1+x^{2}\right) \quad \text { and } \quad K_{\mathrm{ex}}(t)=\exp (t), \quad x \in[0,1], \quad t \in[0,1] .
$$

The functions $f$ and $\beta$ are given by $f(s)=s+1$ and $\beta(s)=s+1$. Note that $\beta$ is linear. The exact kernel $K_{\mathrm{ex}}$ is compared with the numerical solution for $\tau=2^{-3}, 2^{-5}$ and $\tau=2^{-7}$ in Figure 1(a) The errors $E_{K_{\mathrm{cx}}}(\tau)$ are depicted in Figure 1(b), where the error $\log _{2} E_{K_{\mathrm{ex}}}$ is plotted as a function of $\log _{2} \tau$. The linear regression line through all data points is given by $\log _{2} E_{K_{\text {ex }}}=2.0233 \log _{2} \tau-0.9666$. This is in accordance with the predicted convergence rate in Corollary 7.1. The linear regression line for the relative error is given by $\log _{2} E_{K_{\mathrm{ex}}}^{r}=2.0233 \log _{2} \tau-2.6422$. For instance, the CPU time for $\tau=2^{-8}$ is $8 s$. 


\subsection{Experiment 2}

In the second experiment the unknown kernel is sinusoidal, i.e.

$$
u_{\mathrm{ex}}(x, t)=\left(1+t+t^{2}+t^{3}\right)(1+\sin (\pi x)) \quad \text { and } \quad K_{\mathrm{ex}}(t)=\sin (2 \pi t), \quad x \in[0,1], \quad t \in\left[0, \frac{1}{2}\right] .
$$

The functions $f$ and $\beta$ are given by respectively $f(s)=s+5$ and $\beta(s)=s^{2}+s$. The function $\beta$ can be linearized outside the range of $u_{\mathrm{ex}}$. The CPU time for $\tau=2^{-8}$ is $8 s$. The results of the numerical experiment are depicted in Figures 2(a) 2(b) The linear regression line through the data points is given by $\log _{2} E_{K_{\mathrm{ex}}}=2.0282 \log _{2} \tau+0.0166$. The linear regression line for the relative error is given by $\log _{2} E_{K_{\mathrm{ex}}}^{r}=2.0282 \log _{2} \tau+2.0166$. The CPU time for $\tau=2^{-8}$ is $3 s$.

\subsection{Experiment 3}

In the third experiment, the prescribed solution $u$ is more general, i.e.

$$
u_{\mathrm{ex}}(x, t)=\left(1+t+t^{2}+t^{3}\right)(1+\sin (t x)) \quad \text { and } \quad K_{\mathrm{ex}}(t)=\sin (2 \pi t), \quad x \in[1,2], \quad t \in[0,1] .
$$

The functions $f$ and $\beta$ are the same as in Experiment 2. Again, accurate numerical approximations are obtained when the timestep is sufficiently small, see Figures 3(a) 3(b) The linear regression line through all data points is given by $\log _{2} E_{K_{\mathrm{ex}}}=2.0142 \log _{2} \tau+1.8951$. The linear regression line for the relative error is given by $\log _{2} E_{K_{\mathrm{ex}}}^{r}=$ $2.0142 \log _{2} \tau+2.8951$. The CPU time for $\tau=2^{-8}$ is $10 s$.

\section{Conclusion}

A nonlinear parabolic integro-differential problem of second order with an unknown solely time-dependent convolution kernel is considered. The missing information is compensated by an integral-type measurement over the domain. The existence and uniqueness of a weak solution for the problem is proved using Rothe's method. This convergence of the numerical approximations to the exact solution is optimal in time. Numerical experiments support this theoretically obtained results. For instance, future research can concern the detemination of $K$ when a local measurement $\int_{\Omega} \chi(x) u(x, t) \mathrm{d} x=m(t)$ is considered and the implementation of numerical experiments with noisy data.

\section{Acknowledgement}

The research was supported by the IAP P7/02-project of the Belgian Science Policy. 


\section{Appendix A. Figures}

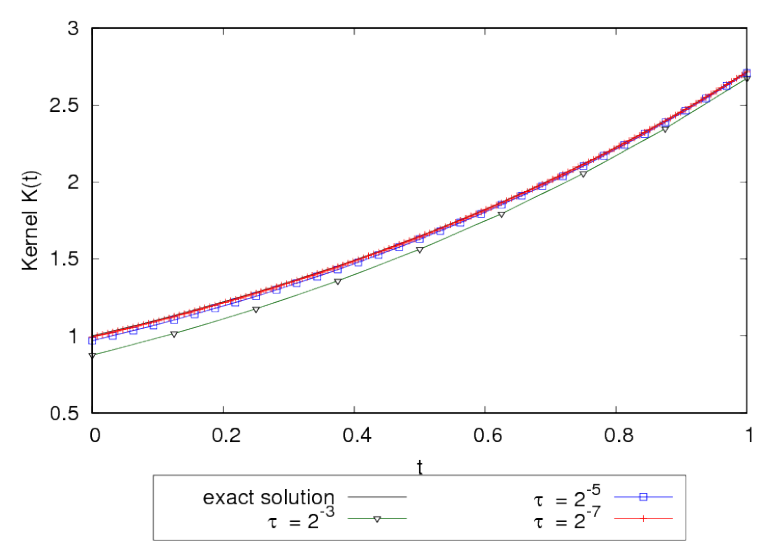

(a) exact solution 29 and its numerical approximation

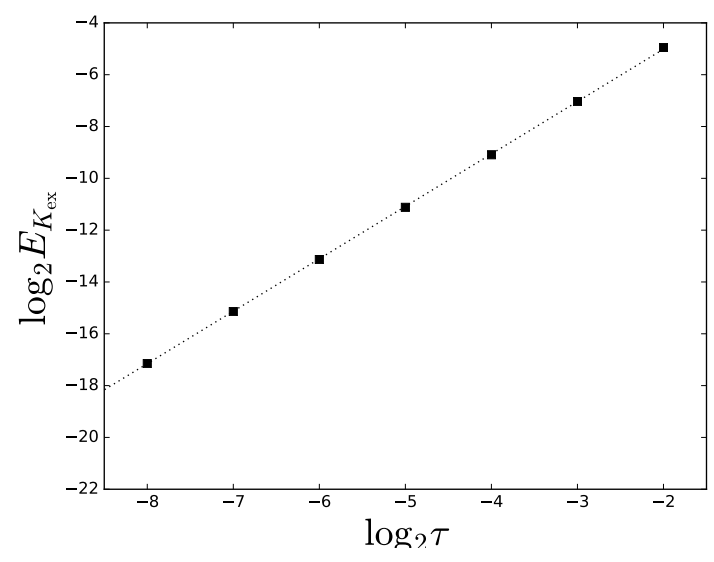

(b) error $E_{K_{\mathrm{ex}}}(\tau)$

Figure A.1. Kernel reconstruction in Experiment 1.

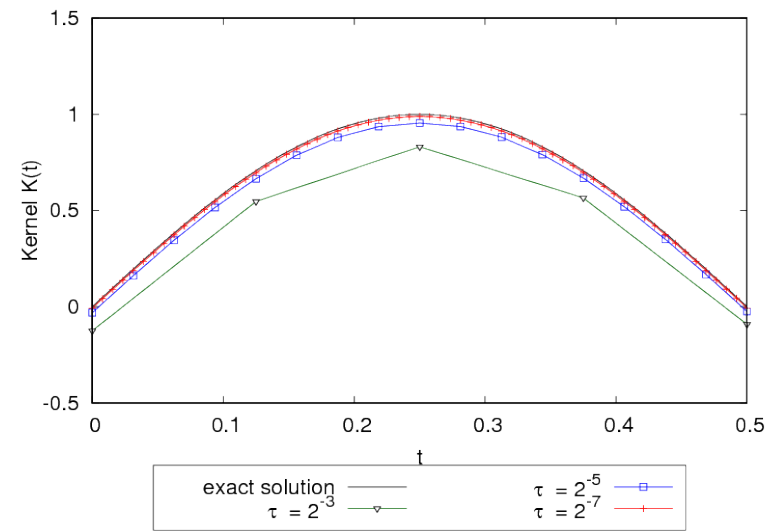

(a) exact solution 30 and its numerical approximation

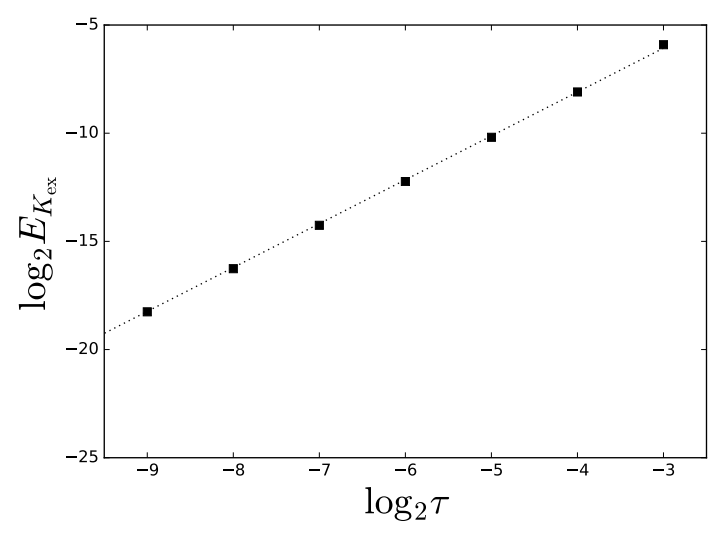

(b) error $E_{K_{\mathrm{ex}}}(\tau)$

Figure A.2. Kernel reconstruction in Experiment 2. 


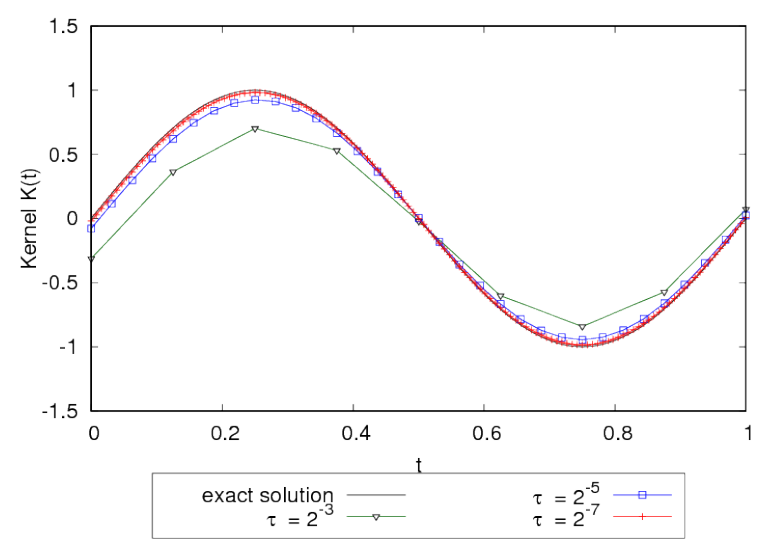

(a) exact solution 31 and its numerical approximation

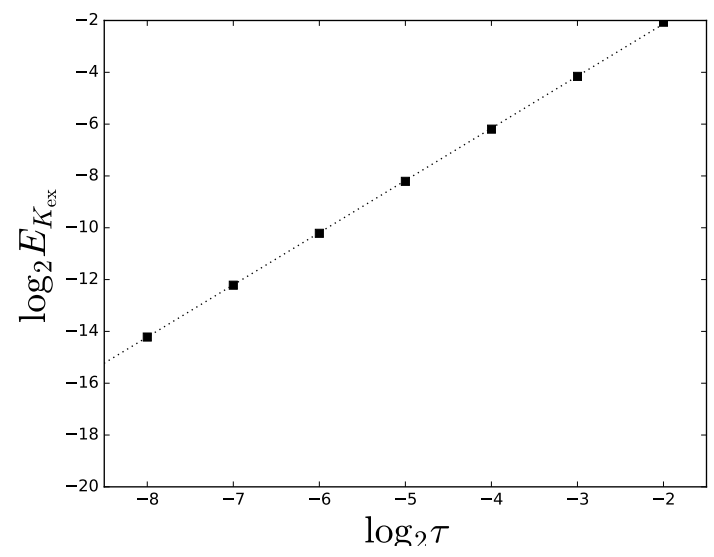

(b) error $E_{K_{\mathrm{ex}}}(\tau)$

Figure A.3. Kernel reconstruction in Experiment 3.

\section{References}

[1] F. Colombo, D. Guidetti, A. Lorenzi, On applications of maximal regularity to inverse problems for integrodifferential equations of parabolic type, Ruiz Goldstein, Gisèle (ed.) et al., Evolution equations. Proceedings of the conference, Blaubeuren, Germany, June 11-17, 2001 in honor of the 60th birthdays of Philippe Bénilan, Jerome A. Goldstein and Rainer Nagel. New York, NY: Marcel Dekker. Lect. Notes Pure Appl. Math. 234, 77-89 (2003).

[2] F. Colombo, D. Guidetti, V. Vespri, Some global in time results for integrodifferential parabolic inverse problems, Favini, Angelo (ed.) et al., Differential equations. Inverse and direct problems. Papers of the meeting, Cortona, Italy, June 21-25, 2004. Boca Raton, FL: CRC Press. Lecture Notes in Pure and Applied Mathematics 251, 35-58 (2006).

[3] F. Colombo, D. Guidetti, A global in time existence and uniqueness result for a semilinear integrodifferential parabolic inverse problem in sobolev spaces, Math. Models Methods Appl. Sci. 17 (4) (2007) 537-565.

[4] D. Guidetti, Convergence to a stationary state for solutions to parabolic inverse problems of reconstruction of convolution kernels., Differ. Integral Equ. 20 (9) (2007) 961-990.

[5] F. Colombo, D. Guidetti, Some results on the identification of memory kernels, in: M. Ruzhansky, J. Wirth (Eds.), Modern aspects of the theory of partial differential equations. Including mainly selected papers based on the presentations at the 7th international ISAAC congress, London, UK, July 13-18, 2009., Operator Theory: Advances and Applications 216. Basel: Birkhäuser, 2011, pp. 121-138.

[6] A. L. Bukhgeǐm, G. V. Dyatlov, Inverse problems for equations with memory, in: Inverse problems and related topics. Proceedings of a seminar, Kobe Institute, Kobe, Japan, February 6-10, 1998, Boca Raton, FL: Chapman \& Hall/CRC, 2000, pp. 19-35.

[7] M. Grasselli, S. Kabanikhin, A. Lorenzi, An inverse hyperbolic integrodifferential problem arising in geophysics. i, Siberian Mathematical Journal 33 (3) (1992) 415-426.

[8] M. Grasselli, An identification problem for an abstract linear hyperbolic integrodifferential equation with applications, Journal of Mathematical Analysis and Applications 171 (1) (1992) 27-60.

[9] C. Cavaterra, M. Grasselli, On an inverse problem for a model of linear viscoelastic kirchhoff plate, J. Integral Equations Applications 9 (3) (1997) 179-218.

[10] J. Janno, Discretization and regularization of an inverse problem related to a quasilinear hyperbolic integrodifferential equation, Inverse Problems 13 (3) (1997) 711.

[11] J. Janno, E. Kiss, L. von Wolfersdorf, On tikhonov regularization for identifying memory kernels in heat conduction and viscoelasticity, ZAMM - Journal of Applied Mathematics and Mechanics / Zeitschrift für Angewandte Mathematik und Mechanik 80 (4) (2000) $259-272$.

[12] R. H. De Staelen, M. Slodička, Reconstruction of a convolution kernel in a semilinear parabolic problem based on a global measurement, Nonlinear Analysis: Theory, Methods \& Applications 112 (2015) 43-57.

[13] R. H. De Staelen, K. Van Bockstal, M. Slodička, Error analysis in the reconstruction of a convolution kernel in a semilinear parabolic problem with integral overdetermination, J. Comput. Appl. Math. 275 (2015) 382-391.

[14] K. Van Bockstal, R. H. De Staelen, M. Slodička, Identification of a memory kernel in a semilinear integrodifferential parabolic problem with applications in heat conduction with memory, J. Comput. Appl. Math. 289 (0) (2015) 196-207.

[15] R. H. De Staelen, Guidetti, On a finite difference scheme for an inverse integro-differential problem using semigroup theory: A functional analytic approach, Numerical Functional Analysis and Optimization 37 (7) (2016) 850-886.

[16] A. Lorenzi, Determination of a time-dependent coefficient in a quasi-linear parabolic equation, Ric. Mat. 32 (2) (1983) $263-284$.

[17] M. Dehghan, Parameter determination in a partial differential equation from the overspecified data, Math. Comput. Model. 41 (2-3) (2005) $196-213$. 
[18] M. Dehghan, Identification of a time-dependent coefficient in a partial differential equation subject to an extra measurement, Numer. Meth. Part. D. E. 21 (3) (2005) 611-622.

[19] A. Saadatmandi, M. Dehghan, Computation of two time-dependent coefficients in a parabolic partial differential equation subject to additional specifications, Int. J. Comput. Math. 87 (5) (2010) 997-1008.

[20] U. M. Fedus', Identification of the coefficient of the time derivative in a quasilinear parabolic equation, Journal of Mathematical Sciences 168 (4) (2010) 523-543.

[21] M. Lakestani, M. Dehghan, The use of chebyshev cardinal functions for the solution of a partial differential equation with an unknown time-dependent coefficient subject to an extra measurement, J. Comput. Appl. Math. 235 (3) (2010) 669-678.

[22] A. Mohebbi, M. Dehghan, High-order scheme for determination of a control parameter in an inverse problem from the over-specified data, Computer Physics Communications 181 (12) (2010) 1947-1954.

[23] M. Ismailov, F. Kanca, D. Lesnic, Determination of a time-dependent heat source under nonlocal boundary and integral overdetermination conditions, Appl. Math. Comput. 218 (8) (2011) 4138-4146.

[24] M. Ismailov, F. Kanca, The inverse problem of finding the time-dependent diffusion coefficient of the heat equation from integral overdetermination data, Inverse Probl. Sci. Eng. 20 (2012) 463-476.

[25] D. Lesnic, S. A. Yousefi, M. Ivanchov, Determination of a time-dependent diffusivity from nonlocal conditions, J. Appl. Math. Comput. 41 (2013) 301-320.

[26] A. Hazanee, D. Lesnic, Determination of a time-dependent coefficient in the bioheat equation, International Journal of Mechanical Sciences 88 (0) (2014) $259-266$.

[27] K. Van Bockstal, M. Slodička, Determination of a time-dependent diffusivity in a nonlinear parabolic problem, Inverse Problems in Science and Engineering 23 (2) (2015) 307-330.

[28] M. Hussein, D. Lesnic, Identification of the time-dependent conductivity of an inhomogeneous diffusive material, Applied Mathematics and Computation 269 (2015) 35-58.

[29] J. W. Delleur, The Handbook of Groundwater Engineering, CRS Press, 1999.

[30] R. C. MacCamy, A model for one-dimensional, nonlinear viscoelasticity, Q. Appl. Math. 35 (1977) 21-33.

[31] I. Neuweiler, D. Erdal, M. Dentz, A non-local Richards equation to model unsaturated flow in highly heterogeneous media under nonequilibrium pressure conditions, Vadose Zone Journal 11 (3) (2012) doi:10.2136/vzj2011.0132.

[32] H. Gajewski, K. Gröger, K. Zacharias, Nichtlineare Operatorgleichungen und Operatordifferentialgleichungen., Mathematische Lehrbücher und Monographien. II. Abteilung. Band 38. Berlin: Akademie-Verlag, 1974.

[33] M. Slodička, S. Dehilis, A nonlinear parabolic equation with a nonlocal boundary term, Journal of Computational and Applied Mathematics 233 (12) (2010) 3130-3138, finite Element Methods in Engineering and Science (FEMTEC 2009).

[34] J. Kačur, Method of Rothe in evolution equations, Vol. 80 of Teubner Texte zur Mathematik, Teubner, Leipzig, 1985.

[35] M. M. Vainberg, Variational method and method of monotone operators in the theory of nonlinear equations. translated from Russian by A. Libin. translation edited by D. Louvish., A Halsted Press Book. New York-Toronto: John Wiley \& Sons; Jerusalem- London: Israel Program for Scientific Translations. xi, 356 p. (1973) (1973).

[36] D. Bainov, P. Simeonov, Integral inequalities and applications, Mathematics and Its Applications. East European Series. 57. Dordrecht, Kluwer Academic Publishers, 1992.

[37] J. Nečas, Les méthodes directes en théorie des équations elliptiques (Direct Methods in the Theory of Elliptic Equations), Academia, Prague, 1967.

[38] R. B. Guenther, J. W. Lee, Partial Differential Equations of Mathematical Physics and Integral Equations, Dover books on mathematics, Dover Publications, United States of America, 1988.

[39] L. C. Evans, Partial differential equations, Vol. 19 of Graduate Studies in Mathematics, Providence, RI: American Mathematical Society, USA, 1998.

[40] A. Kufner, O. John, S. Fučík, Function Spaces, Monographs and textbooks on mechanics of solids and fluids, Noordhoff International Publishing, Leyden, 1977.

[41] W. Rudin, Real and complex analysis, McGraw-Hill, 1987.

[42] A. Logg, G. N. Wells, DOLFIN: Automated Finite Element Computing, ACM Trans. Math. Software 37 (2) (2010) 28.

[43] A. Logg, G. Wells, J. Hake, DOLFIN: a C++/Python Finite Element Library, Springer, Berlin, Heidelberg, 2012 , Ch. 10.

[44] A. Logg, K.-A. Mardal, G. N. Wells, et al., Automated Solution of Differential Equations by the Finite Element Method, Springer, Berlin, Heidelberg, 2012.

[45] M. S. Alnæs, J. Blechta, J. Hake, A. Johansson, B. Kehlet, A. Logg, C. Richardson, J. Ring, M. E. Rognes, G. N. Wells, The FEniCS project version 1.5, Archive of Numerical Software 3 (100). 